\title{
Track Everything: Limiting Prior Knowledge in Online Multi-Object Recognition
}

\author{
Sebastien C. Wong* ${ }^{*}$ Senior Member, IEEE, Victor Stamatescu ${ }^{\dagger}$, Member, IEEE, Adam Gatt ${ }^{\ddagger}$, Member, IEEE, \\ David Kearney $^{\dagger}$, Ivan Lee ${ }^{\dagger}$ Senior Member, IEEE and Mark D. McDonnell ${ }^{\dagger}$, Senior Member, IEEE * Defence \\ Science and Technology Group, Edinburgh, SA, Australia ${ }^{\dagger}$ Computational Learning Systems Laboratory, School \\ of Information Technology and Mathematical Sciences, University of South Australia, Mawson Lakes, SA, \\ Australia $\ddagger$ Australian Defence Force, Edinburgh, SA, Australia
}

\begin{abstract}
This paper addresses the problem of online tracking and classification of multiple objects in an image sequence. Our proposed solution is to first track all objects in the scene without relying on object-specific prior knowledge, which in other systems can take the form of hand-crafted features or user-based track initialization. We then classify the tracked objects with a fastlearning image classifier that is based on a shallow convolutional neural network architecture and demonstrate that object recognition improves when this is combined with object state information from the tracking algorithm. We argue that by transferring the use of prior knowledge from the detection and tracking stages to the classification stage we can design a robust, general purpose object recognition system with the ability to detect and track a variety of object types. We describe our biologically inspired implementation, which adaptively learns the shape and motion of tracked objects, and apply it to the Neovision2 Tower benchmark data set, which contains multiple object types. An experimental evaluation demonstrates that our approach is competitive with state-of-the-art video object recognition systems that do make use of object-specific prior knowledge in detection and tracking, while providing additional practical advantages by virtue of its generality.
\end{abstract}

Index Terms-object recognition, image classification, visual tracking, multi-object tracking

\section{INTRODUCTION}

We report on the design of an automated vision system that can accurately locate and recognize multiple types of objects. The goal of online object recognition systems is to continuously detect and correctly classify the objects in a scene as they undergo changes in motion or appearance. Furthermore, the system should be robust to distracting or occluding clutter. Our proposed solution to these challenges is an adaptive multiple object tracking (MOT) algorithm that tracks all objects in the scene and defers any decisions on what is an object of interest to a separate classification stage. Object recognition then involves combining these class predictions, with state information given by object tracking. This approach emulates the separate what and where processing streams in primate vision [1], and allows the tracking process to be performed without any reliance on object-specific prior knowledge.

Manuscript received September 26, 2016, accepted April 9 2017. Corresponding authors: S. C. Wong (email: sebastien@computer.org), V. Stamatescu (email: victor.stamatescu@unisa.edu.au)
An important practical consideration in the design of online object recognition systems is the finite amount of labeled and annotated data available for training. When scarce, this can degrade classification performance due to overfitting and reduce the detection probability of highly tuned object detectors. Even when larger data sets are available, these may be biased in such a way that their image statistics do not accurately reflect the data encountered by the system at run time [2]. In the case of classifier-based object recognition [3] and detection [4], the use of features, which are higher-level representations of an object than the raw image, can mitigate these problems by providing a degree of invariance across different data sets. In the case of tracking and object detection algorithms, the same set of challenges can be addressed by making the tracker and detector designs less domain-specific. In our system this is achieved through the use of adaptive tracking (e.g. [5], [6]) and by employing a track-before-detect [7] approach that delays the requirement for object specific prior knowledge from detection until recognition.

We note that there exist commercial and security video analysis applications in which the user may not possess specific knowledge about new, previously unseen objects. For example, the user may not have access to information on the appearance of a set of target objects, but may still wish to track these targets in order to accumulate a domain-specific data set. Moreover, it may be impractical for the user to initialize the system on multiple targets, especially when more objects are expected to come into view, or are stationary for long periods. Therefore, in applications where the system requirements are initially not well defined, a useful first step is for the system to autonomously detect and track all (moving and stationary) objects, including those that may, at first, not be considered objects of interest.

Given these aims and real-world requirements, we present a novel approach to online object recognition centered on the idea of tracking all salient objects in the scene. We argue that this "track everything" approach can be realized by limiting the explicit use of prior knowledge, and demonstrate that this can be implemented by simultaneously learning both feature and spatial information about each object and assigning new measurements to system tracks. This argument is supported by the following contributions:

- a novel object shape learning algorithm, the Shape Estimating Filter (SEF), and its multi-object counterpart, the 
Competitive Attentional Correlation Tracker using Shape (CACTuS) [8];

- the integration of a feature learning (FL) algorithm with a shape learning algorithm [9];

- CACTuS-FL: the first algorithm to automatically detect and track multiple objects in a video sequence without object-specific prior knowledge [10];

- an online object recognition system that employs an ensemble of single hidden layer feedforward networks (SLFNs) to combine state information from the multiobject tracking algorithm (CACTuS-FL) with the output from an image classifier, the Shallow Convolutional Neural Network (S-CNN).

The rest of this paper is organized as follows. Key recent advances in the areas of multi-object tracking, image classification and object recognition systems are outlined in Section An overview of our system is provided in Section [II, and this is expanded upon in Sections IV to VI We demonstrate and examine the efficacy of our approach using Neovision2 benchmark data in Section VII. Finally, Section VIII concludes the paper with a summary of our findings.

\section{RELATED WORK}

We review related works in the areas of online multi-object detection and tracking, object recognition, and benchmarks for evaluating such systems.

Online detection and tracking: Recent state-of-the-art online multi-object trackers (e.g. [11], [12], [13], [14], [15]) follow the tracking-by-detection approach, where objects of interest are detected independently in each frame and then uniquely associated with system tracks from the previous frame. The term online implies that the underlying algorithm may only use information collected up to the current frame. The aforementioned examples rely on specialised people detectors, with the exception of Urban Tracker [15], which uses background subtraction to detect all types of traffic under the assumption that only moving objects are of interest. This assumption of motion can also be used to form tracklets [16], elementary trajectory fragments, which can clustered together (usually in an off-line manner) to form complete tracks. Although tracking-by-detection algorithms are state-of-the-art, one limitation stems from noisy or missed detections, which can lead to incomplete system tracks. New systems generally aim to mitigate this problem through more reliable object detector design and/or better data association techniques. For example, Breitenstein et al. [12] handled occlusions by coupling detection confidence maps with an association scheme based on online-learned classifiers. Bae \& Yoon [14] used tracklet confidence to resolve unreliable detections, while their data association stage was based on online discriminative appearance learning. Unlike the aforementioned examples, our system relies instead on the track-before-detect paradigm [7], which is less prone to missing weak detections. Under this approach, the tracking process guides the detection process in order to correlate detections over multiple frames.

Recognition: Our approach to object recognition is motivated by the success of deep learning for image classification tasks (see [17] for a recent review). This typically involves training deep (multi-layered) hierarchical models such as Deep Belief Networks (DBNs) [18] and Convolutional Neural Networks (CNNs) [19]. By training complex models with large amounts of data CNNs have set new image classification benchmarks in recent years through models such as AlexNet [20], OverFeat [21] and VGGNet [22]. Rather than relying on such deep architectures, however, our system performs object recognition using a Shallow CNN [23] that limits learning to a single layer. It has been shown to achieve competitive results on standard image classification data sets [24] while being fast to train (when compared with standard deep learning approaches) and maintaining low implementation complexity (few tuneable metaparameters).

Benchmark data: The third key ingredient to our system is domain-specific image sequence data with sufficient object class labeled examples to allow the supervised training of S-CNNs. As mentioned previously, most public multi-object tracking data sets, including those collected for the recent MOT Challenge [25], contain only a single (pedestrian) target class. This focus on people tracking is highlighted by the latest data release, MOT16 [26], in which ground truth object classes are grouped into three broad categories: Target (pedestrian, cyclist, skater), Ambiguous (lying/sitting person, reflection, distractor), Other (car, motorbike, occluder, bicycle). An image sequence data set that does contain multiple object types has been provided by the DARPA Neovision2 [27] program. This data set was collected to enable training and evaluation of Neuromorphic Vision algorithms [28], [29], [30], [31], which are a class of object recognition algorithms motivated by the emergence of bio-inspired vision sensors [32] and processing hardware (e.g. [33]).

Prior knowledge: As previously discussed, in a trackingby-detection approach [11], [12], [13], [14] object specific prior knowledge is embedded into the detector model. Another common prior assumption is that only moving objects are of interest, leading to detection through background subtraction [15], or track formation through tracklets [16]. These assumptions limit tracking to only a specific set of objects, or only moving objects. Furthermore, offline trackers not only make use of prior knowledge of objects, but also incorporate knowledge about future frames, and thus can not run on streaming video. For object recognition using a CNN [22], [24] prior knowledge is strongly embedded into these models through the large training data sets. Thus, there is sufficient scope within the literature to investigate an online system design that transfers prior knowledge from detection and tracking into recognition.

\section{OVERVIEW}

This section provides an overview of our online object recognition system, shown in Figure 1, as well the notation used in this paper.

Road map: Section IV describes the generic feature extraction stage that is used by the what and where processing streams. The where processing stream (Section V) seeks to locate salient objects in the scene and guide the attention of the what processing stream (Section VI) to these objects. 


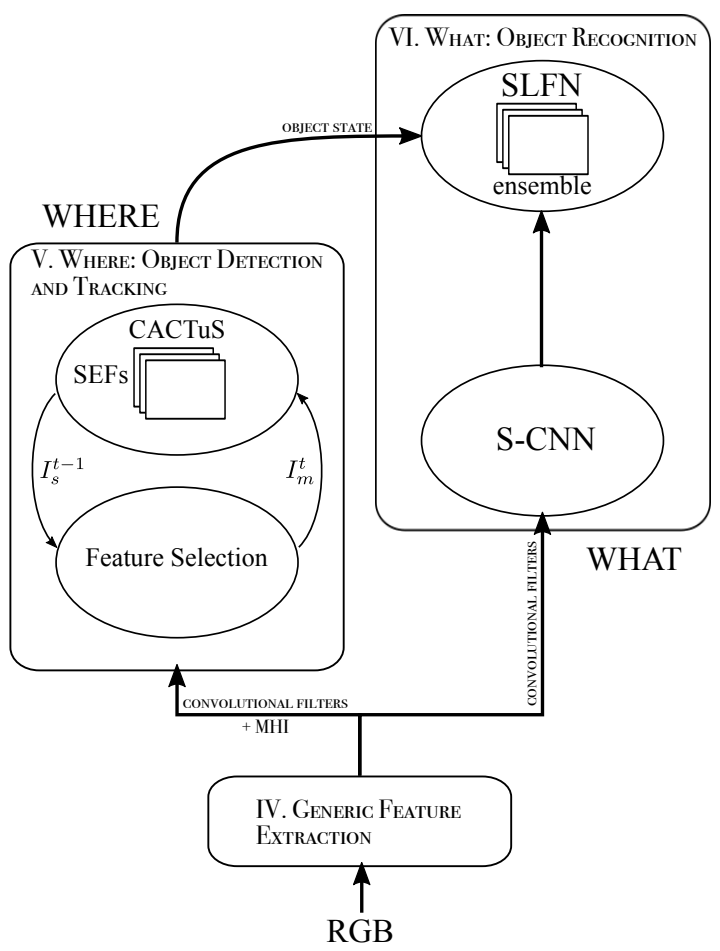

Fig. 1. Overview of our system for online object recognition comprising the where (CACTuS-FL) and what (S-CNN and SLFN ensemble) processing streams. The SLFN also combines object state information from the where stream.

The where stream is handled by the autonomous multi-object tracking algorithm CACTuS-FL [10]. The what processing stream relies on a S-CNN architecture [23] that is followed by an ensemble of SLFNs [24], which combines the S-CNN output with object state information from the where processing stream. The S-CNN and individual SLFNs are trained offline and then deployed in the online classification of image regions (or patches) associated with system tracks.

Notation: Probability mass functions (PMFs) are denoted by capital letters. The subscripts $p, m, \& s$ are used to denote predicted, measured and posterior PMFs respectively, while the subscript 0 denotes a constant prior. The superscripts $t$ and $t-1$ denote the current and previous time frames respectively. For brevity, equations that operate only on the current frame do not include superscript $t$. The notation for normalizing across all bins $\boldsymbol{u}$ of a histogram to form a PMF is abbreviated to $\frac{1}{\Sigma_{u}}$ to avoid additional indexing variables.

\section{Generic Feature Extraction}

Good features are those which provide a response that discriminates the object(s) of interest and is invariant to changes in the scene. Here we desire a set of common features that are good for both detection and recognition. Furthermore, for our track everything approach every candidate object (including clutter and stationary objects) should be tracked, and is therefore of interest.

Our tracker, CACTuS-FL, can operate on any set of features, including hand-crafted features [10], however, recent experimental evidence demonstrates that convolutional filters learned by CNNs can produce good features for online visual tracking, enhancing state-of-the-art performance [34], [35]. Furthermore, while motion provides a strong visual cue to the presence of salient objects, which can form an image feature [36] or constrain appearance models [37], this type of cue can not, by itself, detect stationary objects.

For object recognition, the orderless pooling of CNN filter banks can also provide state-of-the-art performance [38], despite earlier evidence to the contrary [39].

Thus, we choose a motion history image (MHI) feature [36], as moving (as well as stationary) objects are of interest, and a biologically inspired convolutional filter bank [40] that is learned in a generative manner to encapsulate the entire scene.

\section{A. Motion History Image}

The MHI [36] combines object movement information over an image sub-sequence. To meet the requirement of online tracking we avoid the backward MHI and implement only the forward MHI. This candidate feature is obtained from frame differences between the current image and historical images (through a Markov chain), which highlights the cumulative object motion with a gradient trail that fades away.

\section{B. Convolutional Filters}

The 24 convolutional filters, shown in Figure 2, were learned in an unsupervised manner from the first frames of Neovision2 Tower training image sequences $010-024$ by using a Convolutional Restricted Boltzmann Machine (CRBM) [41]. Each greyscale filter has dimensions of $16 \times 16$ pixels, which was chosen empirically [40]. In training the generative CRBM model, RGB input images were first downsampled by a factor of two (to a size of $960 \times 540$ pixels) to match the resolution of input images used in the online object recognition system. The training images were pre-processed by converting to greyscale, applying the whitening function used by Olshausen \& Field [42], subtracting the image mean and normalizing the result by its root mean square $(\mathrm{rms})$, as illustrated in Figure 3. The whitening function applies a combined whitening and low-pass filter with frequency response of the form $f e^{-\left(f / f_{0}\right)^{4}}$, where $f_{0}$ is a cutoff frequency of 200 cycles/image. During the online application of these filters, each new input image also undergoes these pre-processing steps.

\section{Where: OBJECt Detection AND TRACKING}

Multiple object tracking algorithms are required to maintain temporally consistent trajectories (state information) for all objects and to uniquely associate new observations with each trajectory. An additional requirement in our design is that tracks are able to self-initialize by automatically converging onto regions of temporally consistent and spatially correlated local saliency. To this end, we couple the track-before-detect paradigm with an adaptive tracking approach (e.g. [5], [6]), so that a state model, which recursively learns both object shape and motion, is able to guide future detections. The unique identities of multiple objects are preserved by correctly 


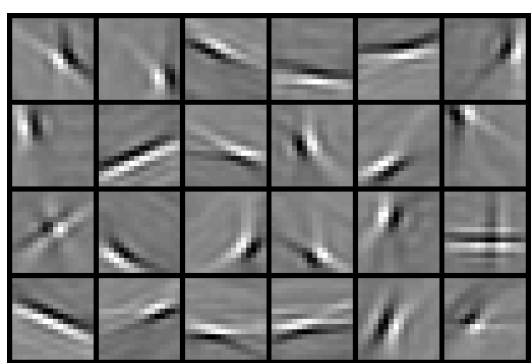

Fig. 2. Bank of 24 generative filters of size $16 \times 16$ pixels learned using a Convolutional Restricted Boltzmann Machine (CRBM) [41]. The unsupervised training was carried out using the first frames of Neovision2 Tower training sequences. All training image were first converted to greyscale and pre-processed (see main text for details).
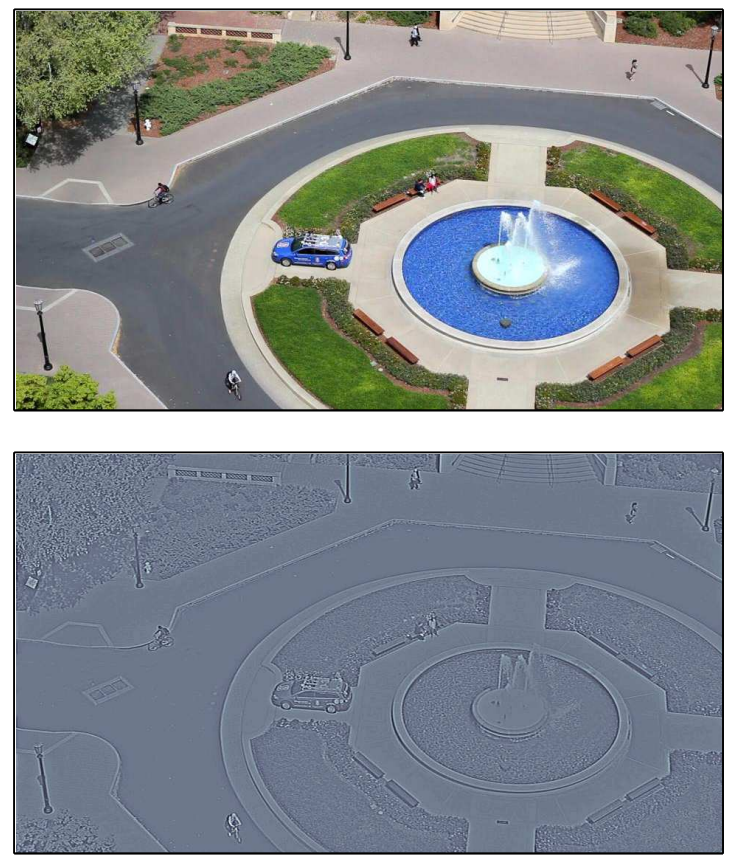

Fig. 3. Sample RGB (top) and pre-processed (bottom) input image, showing frame 61 from Neovision2 [27] Tower image sequence 001, which was first downsampled to a size of $960 \times 540$ pixels. Image pre-processing involves the application of the whitening function used by Olshausen \& Field [42], subtraction of the image mean and normalization of the result by its root mean square $(r m s)$.

associating multiple sub-trackers with new observations. This is accomplished by operating these sub-trackers in competition with one another across the scene.

\section{A. Feature Selection}

We first address the problem of autonomous single object detection. Typical object detectors in visual tracking use application-specific knowledge such as hard-coding a fixed set of features that describe a particular object or type of object. By contrast, this paper follows the adaptive method proposed by Collins et al. [43], which frames the online selection of a subset of features (from a larger set) as an evolving "object versus local background" two-class classification problem.
This discriminant tracking approach is analogous to the centersurround mechanisms for attention and saliency that are found in biological vision [44] and enable automatic track initiation.

Every candidate feature $n \in 1, \ldots, 25$ (the MHI feature and the 24 convolutional features from Section IV is used to compute a feature map $Z_{n}^{t}(\boldsymbol{i})$, which is a representation of the image at frame $t$ in terms of the feature response at each pixel position $\boldsymbol{i}$. Following [43], discriminative features are selected based on the separation of their class-conditioned feature response distributions $F_{n}^{t}(u)$ and $B_{n}^{t}(u)$, which are 1D histograms extracted for each feature from the object foreground and local background regions, respectively. Here $u \in 1, \ldots, 64$ is an index into a histogram of feature response values. In order to extract the object feature response distribution we use the learned object image from the previous frame $I_{\mathrm{s}}^{t-1}$, defined by Eqn. 23, as a pixel weighting mask:

$$
F_{n}^{t}(u)=\frac{\sum_{i} I_{\mathrm{s}}^{t-1}(\boldsymbol{i}) \delta\left(Z_{n}^{t}(\boldsymbol{i})-u\right)}{\Sigma_{u}},
$$

where $\delta$ is the Kronecker delta function. The local background feature response distribution $B_{n}^{t}$ is extracted in a similar way, using a weighting mask $1-I_{\mathrm{s}}^{t-1}$ over an appropriately sized local image patch. Using the learned image $I_{\mathrm{s}}^{t-1}$ to precisely identify object pixels leads to a more precise extraction of the feature response distributions than with a bounding box (as used in [43]), reducing background pollution in the feature learning process [9]. This, in turn, provides stronger detections for the tracking process. This feedback between tracking and feature selection is illustrated in Figure 1

A detection map $\hat{L}_{n}(\boldsymbol{i})$ is computed for each feature by back-projecting its Likelihood Ratio $L_{n}(u)=$ $F_{n}^{t}(u) / B_{n}^{t}(u)$ into its feature map and normalizing: $\hat{L}_{n}(\boldsymbol{i})=$ $L_{n}\left(u=Z_{n}^{t}(\boldsymbol{i})\right) / \max \left(L_{n}\left(u=Z_{n}^{t}(\boldsymbol{i})\right)\right)$, see [43] for the original formulation and [9] for an illustrated example. Online feature selection then involves choosing the most discriminable set of $N$ detection maps, with $N=6$ chosen empirically, as similar values $(4-8)$ yielded comparable tracking performance. By considering the feature response in each pixel of a local image region (i.e. object, local background, or both) as a discrete random variable $\boldsymbol{z}_{n}^{t}$, we use Maximum Marginal Diversity (MMD) [45] to approximate the infomax space: the subset of $N$ features that maximizes its own mutual information with the class label random variable $c$. When applied to feature selection in discriminant tracking [44], [46] MMD involves scoring each feature by its mutual information $\mathcal{I}\left(\boldsymbol{z}_{n}^{t} ; \boldsymbol{c}\right)$ with the object $(\boldsymbol{c}=1)$ and local background $(\boldsymbol{c}=0)$ class labels:

$$
\mathcal{I}\left(\boldsymbol{z}_{n}^{t} ; \boldsymbol{c}\right)=\sum_{c=0}^{1} p(\boldsymbol{c}=c) \mathcal{R}\left[p\left(\boldsymbol{z}_{n}^{t}=u \mid \boldsymbol{c}=c\right) \| p\left(\boldsymbol{z}_{n}^{t}=u\right)\right]
$$

where $\mathcal{R}[p(u) \| q(u)]=\sum_{u \in U} p(u) \log _{2} \frac{p(u)}{q(u)}$ is the KullbackLeibler divergence between two distributions $p$ and $q$. Here the class-conditioned feature response distributions $p\left(\boldsymbol{z}_{n}^{t}=u \mid \boldsymbol{c}=\right.$ 1) and $p\left(\boldsymbol{z}_{n}^{t}=u \mid \boldsymbol{c}=0\right)$ are given by $F_{n}^{t}(u)$ and $B_{n}^{t}(u)$, respectively, while $p\left(\boldsymbol{z}_{n}^{t}=u\right)$ corresponds to the combined object and local background regions.

The most discriminative detection maps are selected by choosing the $N$ highest scores given by Eqn. (2), and these 
are summed pixel-wise in a weighted average to produce a fused detection map $I_{\mathrm{m}}^{t}$ that serves as input to the tracking algorithm:

$$
I_{\mathrm{m}}^{t}(\boldsymbol{i})=\sum_{n=1}^{N} w_{n} \hat{L}_{n}(\boldsymbol{i}) .
$$

The weights in Eqn. (3) are given by $w_{n}=\mathrm{I}\left(\boldsymbol{z}_{n}^{t} ; \boldsymbol{c}\right) \times B$, where the similarity score $\bar{B}$ is the Bhattacharyya coefficient [47]:

$$
B=\sum_{u} \sqrt{F_{n, \mathrm{~m}}^{t}(u) F_{n, \mathrm{~s}}^{t-1}(u)},
$$

which rewards temporal consistency between the object feature response $F_{n, \mathrm{~m}}^{t}$ measured in the current frame according to Eqn. (1) and an object feature response learned up to the previous frame $F_{n, \mathrm{~s}}^{t-1}$. The learned posterior feature response $F_{n, \mathrm{~s}}^{t}$ is updated at each frame by

$$
F_{n, \mathrm{~s}}^{t}(u)=\frac{F_{n, \mathrm{~s}}^{t-1}(u) F_{n, \mathrm{~m}}^{t}(u)}{\Sigma_{u}} .
$$

\section{B. Shape Estimating Filters}

We next address the problem of adaptively learning an object state model, which includes a probabilistic representation of its shape. The proposed solution is a single object tracker called the Shape Estimating Filter (SEF) [8], which combines spatiotemporal information from past frames with new measurements to recursively estimate the object position, velocity and shape. A SEF autonomously correlates recurring saliency from each new fused detection map into shape and trajectory estimates.

Assuming that only a single object is present in an image, the 2D PMF $I(\boldsymbol{i})$ is used to describe the probability that a given pixel $\boldsymbol{i}=\left(i_{1}, i_{2}\right)$ belongs to that object. The PMF $I(\boldsymbol{i})$ can then be factored into 2D PMFs for shape $S(\boldsymbol{j})$ and position $X(\boldsymbol{x})$. Here $X(\boldsymbol{x})$ represents the probability that the object center of mass has position $\boldsymbol{x}=\left(x_{1}, x_{2}\right)$, while $S(\boldsymbol{j})$ is proportional to the probability that the pixel $j=\left(j_{1}, j_{2}\right)$ is part of the object. The vectors $\boldsymbol{i}, \boldsymbol{j}$ and $\boldsymbol{x}$ are considered 2D random variables operating on the set of integers. The relationship between image, position and shape random variables is given by $\boldsymbol{i}=\boldsymbol{x}+\boldsymbol{j}$, which can be expressed as $\boldsymbol{x}=\boldsymbol{i}-\boldsymbol{j}$, or as $\boldsymbol{j}=\boldsymbol{i}-\boldsymbol{x}$. This relationship allows the shape of an object to be decoupled from its position in the image.

In order to describe the object motion across a sequence of images in an adaptive manner, 2D random variables are used to model acceleration $\boldsymbol{a}$ and velocity $\boldsymbol{v}$. These variables are described by the PMFs $A(\boldsymbol{a})$ and $V(\boldsymbol{v})$, respectively. Assuming a simplified Euler motion (non-rotational pointmass) for the object and that $\Delta t=t-(t-1)=1$ leads to the following relationships: $\boldsymbol{v}^{t}=\boldsymbol{v}^{t-1}+\boldsymbol{a}^{t}$ and $\boldsymbol{x}^{t}=\boldsymbol{x}^{t-1}+\boldsymbol{v}^{t}$. Rearranging, this gives: $\boldsymbol{v}^{t}=\boldsymbol{x}^{t}-\boldsymbol{x}^{t-1}$ and $\boldsymbol{a}^{t}=\boldsymbol{v}^{t}-\boldsymbol{v}^{t-1}$.

To handle deformable objects, the 2D PMF $R(\boldsymbol{r})$ is defined as the change in shape from one frame to the next, which is described by the random variable relationship $r=j^{t}-j^{t-1}$.

These random variable relationships are used to build the SEF algorithm, using the operations of convolution $\otimes$ and cross-correlation $\hat{\otimes}$, as illustrated in Figure 4 . The SEF statespace hierarchy provides a framework for combining top-down

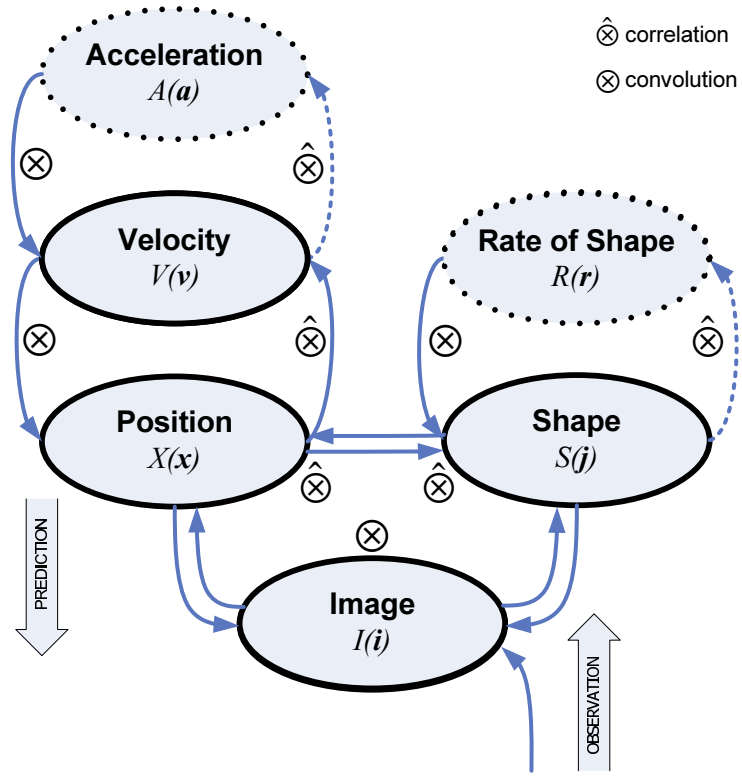

Fig. 4. The hierarchical state model of the Shape Estimating Filter (SEF) [8] Predictions are propagated from the top-down and new observations from the bottom-up. Predictions and observations are combined at each layer to provide an approximate Bayesian update of the state model.

predictions with bottom-up sensory measurements through a Bayesian update process.

Predictions are made by traversing down the state model hierarchy (starting in the top left of Figure 4) according to:

$$
\begin{aligned}
& \boldsymbol{v}^{t}=\boldsymbol{v}^{t-1}+\boldsymbol{a} \quad \Rightarrow \quad V_{\mathrm{p}}^{t}=V_{\mathrm{s}}^{t-1} \otimes A_{0}, \\
& \boldsymbol{x}^{t}=\boldsymbol{x}^{t-1}+\boldsymbol{v}^{t} \quad \Rightarrow \quad X_{\mathrm{p}}^{t}=X_{\mathrm{s}}^{t-1} \otimes V_{\mathrm{p}}^{t} \text {, } \\
& j^{t}=j^{t-1}+\boldsymbol{r} \quad \Rightarrow \quad S_{\mathrm{p}}^{t}=S_{\mathrm{s}}^{t-1} \otimes R_{0} \text {, } \\
& \boldsymbol{i}^{t}=\boldsymbol{j}^{t}+\boldsymbol{x}^{t} \quad \Rightarrow \quad I_{\mathrm{p}}^{t}=S_{\mathrm{p}}^{t} \otimes X_{\mathrm{p}}^{t} \text {, }
\end{aligned}
$$

where $A_{0}$ and $R_{0}$ are 2D Gaussian priors.

Given $I_{\mathrm{m}}^{t}$, measurements are made by traversing up the state model hierarchy (starting at the bottom of Figure 4) according to:

$$
\begin{array}{lll}
\boldsymbol{x}^{t}=\boldsymbol{i}^{t}-\boldsymbol{j}^{t} & \Rightarrow & X_{\mathrm{m}}^{t}=I_{\mathrm{m}}^{t} \hat{\otimes} S_{\mathrm{p}}^{t}, \\
\boldsymbol{v}^{t}=\boldsymbol{x}^{t}-\boldsymbol{x}^{t-1} & \Rightarrow & V_{\mathrm{m}}^{t}=X_{\mathrm{m}}^{t} \hat{\otimes} X_{\mathrm{s}}^{t-1}, \\
\boldsymbol{j}^{t}=\boldsymbol{i}^{t}-\boldsymbol{x}^{t} & \Rightarrow & S_{\mathrm{m}}^{t}=I_{\mathrm{m}}^{t} \hat{\otimes} X_{\mathrm{s}}^{t} .
\end{array}
$$

An approximate Bayesian update scheme is used to combine top-down predictions with bottom-up observations. The posterior PMFs of position, velocity and shape are described by:

$$
\begin{aligned}
X_{\mathrm{s}}^{t}(\boldsymbol{x}) & =\frac{X_{\mathrm{m}}^{t}(\boldsymbol{x}) X_{\mathrm{p}}^{t}(\boldsymbol{x})}{\Sigma_{\boldsymbol{x}}}, \\
V_{\mathrm{s}}^{t}(\boldsymbol{v}) & =\frac{V_{\mathrm{m}}^{t}(\boldsymbol{v}) V_{\mathrm{p}}^{t}(\boldsymbol{v})}{\Sigma_{\boldsymbol{v}}}, \\
S_{\mathrm{s}}^{t}(\boldsymbol{j}) & =\frac{S_{\mathrm{m}}^{t}(\boldsymbol{j}) S_{\mathrm{p}}^{t}(\boldsymbol{j})}{\Sigma_{\boldsymbol{j}}} .
\end{aligned}
$$



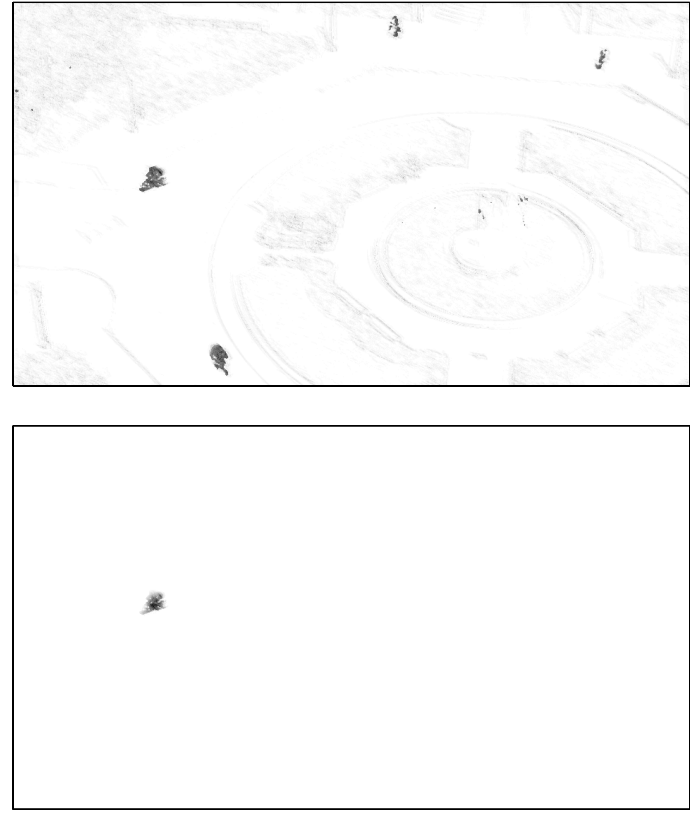

Fig. 5. The selective attentional mechanism of CACTuS-FL, for SEF $k=33$ which is tracking a cyclist, in frame 61 of Neovision 2 Tower image sequence 001. The $I_{\mathrm{m}}^{k}(\boldsymbol{i})$ fused detection map from Eqn. 3 (top) is modulated by the spatial area of attention $\beta^{k}(\boldsymbol{i})$ to form the bottom-up input for the SEF (bottom).

\section{Competitive Attention Correlation Tracking using Shape}

Finally, we address the problem of automatically associating new measurements to multiple system tracks. The proposed solution, which extends the work of Strens and Gregory [48], operates multiple SEFs simultaneously in a competitive attentional framework designed to enforce the tracking of multiple objects. Under this scheme, the SEFs track everything in the scene, including parts of the background or sources of clutter, so that every new measurement is assigned to the SEF that best describes that measurement [10].

For each frame $t$, the multi-object tracking algorithm operates $k=1, \ldots, K$ individual SEFs. The bottom-up input of each SEF $k$ is modulated by an association term $\beta^{k}(i)$, so that Eqn. (3) becomes

$$
I_{\mathrm{m}}^{k}(\boldsymbol{i})=\beta^{k}(\boldsymbol{i}) \sum_{n=1}^{N} w_{n} \hat{L}_{n}(\boldsymbol{i}) .
$$

As shown in Figure 5, top-down modulation provides each SEF with a spatial area of attention to collect new measurements. The term $\beta^{k}(i)$ is computed from learned predictions about the expected image:

$$
\beta^{k}(\boldsymbol{i})=\frac{I_{\mathrm{p}}^{k}(\boldsymbol{i})}{\sum_{j=1}^{K} I_{\mathrm{p}}^{j}(\boldsymbol{i})} .
$$

This selective attentional mechanism modifies the bottom-up input to each SEF, enabling individual SEFs to selectively ignore pixels that are strongly claimed by another SEF, where $0 \leq \beta^{k}(\boldsymbol{i}) \leq 1$ describes the strength of the claim of pixel at location $i$ by SEF $k$.
By assuming a 2D Gaussian prior shape $S_{0}$, an additional attentional mechanism is introduced by replacing Eqn. 10 with

$$
X_{\mathrm{m}}^{k}(\boldsymbol{x})=I_{\mathrm{m}}^{k}(\boldsymbol{i}) \hat{\otimes}\left(S_{\mathrm{p}}^{k}(\boldsymbol{j}) S_{0}(\boldsymbol{j})\right) .
$$

This introduces a self-centering capability to the system [49], which reduces the problem of model drift [50] that affects correlation trackers [6].

In order to encourage SEFs to track multiple objects, Eqn. 13 is modified by a winner-take-more competitive mechanism [48]. Under this scheme, which has the inherent assumption that different objects tend to occupy different positions, $K$ separate SEFs compete over position $\boldsymbol{x}$ to track every object in the scene. Each SEF $k$ competes against all SEFs for its own share of the total association probability $\sum_{l=1}^{K} C^{l}(\boldsymbol{x})=1$ at each position $\boldsymbol{x}$. The individual association probability $C^{k}$, which is shown for a single SEF in Figure 6, is computed using the predicted position $X_{\mathrm{p}}^{k}(\boldsymbol{x})$ according to

$$
C^{k}(\boldsymbol{x})=\frac{X_{\mathrm{p}}^{k}(\boldsymbol{x})}{\sum_{l=1}^{K} X_{\mathrm{p}}^{l}(\boldsymbol{x})} .
$$

The update of position $X_{\mathrm{s}}^{k}(\boldsymbol{x})$ for each SEF $k$ in Eqn. $[13$ is then modified to include this spatial attention modulation for each SEF

$$
X_{\mathrm{s}}^{k}(\boldsymbol{x})=\frac{X_{\mathrm{m}}^{k}(\boldsymbol{x}) X_{\mathrm{p}}^{k}(\boldsymbol{x}) C^{k}(\boldsymbol{x})}{\Sigma_{\boldsymbol{x}}} .
$$

An example of $X_{\mathrm{s}}^{k}(\boldsymbol{x})$ is shown for a single SEF in Figure 6 This mechanism enables the SEF that best describes the position state estimate for a particular object to converge on a region corresponding to that object and exclude other SEFs from that region. This competition encourages SEFs to track different objects, rather than all SEFs converging on the most salient object in the scene.

The shape of the object is observed using the relationship $j=i-x$. First, the best estimate of the object location in the current fused detection map $I_{\mathrm{m}}^{k}(\boldsymbol{i})$ is extracted from the posterior position PMF $X_{\mathrm{s}}^{k}(\boldsymbol{x})$ according to $X_{\mathrm{smax}}^{k}(\boldsymbol{x})=$ $\delta\left(\operatorname{argmax}_{1}\left(\mathrm{X}_{\mathrm{s}}^{\mathrm{k}}(\boldsymbol{x})\right)-\boldsymbol{x}\right)$, where $\operatorname{argmax}_{1}$ returns one maximum. Next, the PMF $X_{\text {smax }}^{k}(\boldsymbol{x})$ is used to extract the observed shape $S_{\mathrm{m}}^{k}(\boldsymbol{j})$ from $I_{\mathrm{m}}^{k}(\boldsymbol{i})$ using:

$$
S_{\mathrm{m}}^{k}(\boldsymbol{j})=I_{\mathrm{m}}^{k}(\boldsymbol{i}) \hat{\otimes} X_{\mathrm{smax}}^{k}(\boldsymbol{x}) .
$$

The process used to update shape has been adapted from [6] as a way to mitigate model drift. First, the degree of match $\rho$ is computed as the $L^{2}$ normalized cross-correlation at $\boldsymbol{j}=(0,0)$ of the measured and predicted shapes, $\rho=$ $\hat{S_{\mathrm{m}}}((0,0)) \hat{\otimes} \hat{S_{\mathrm{p}}}((0,0))$, where $0 \leq \rho \leq 1$ is a scalar.

Next the parameter $\alpha$ is computed as $\alpha(\rho, \lambda)=H(\rho-\lambda) \rho^{2}$, where $H(x)$ is the unit step function and the threshold $\lambda$ acts as the vigilance parameter [51] to ensure that very poor observations are not introduced into memory, see [6] for details.

This controls the degree by which the posterior shape $S_{\mathrm{s}}^{k}(\boldsymbol{j})$ is influenced by new observations $S_{\mathrm{m}}^{k}(\boldsymbol{j})$, or prior expectations $S_{\mathrm{p}}^{k}(\boldsymbol{j})$, and thus Eqn. 15, is replaced with

$$
S_{\mathrm{s}}^{k}(\boldsymbol{j})=\left(S_{\mathrm{m}}^{k}(\boldsymbol{j})\right)^{\alpha}\left(S_{\mathrm{p}}^{k}(\boldsymbol{j})\right)^{(1-\alpha)} .
$$



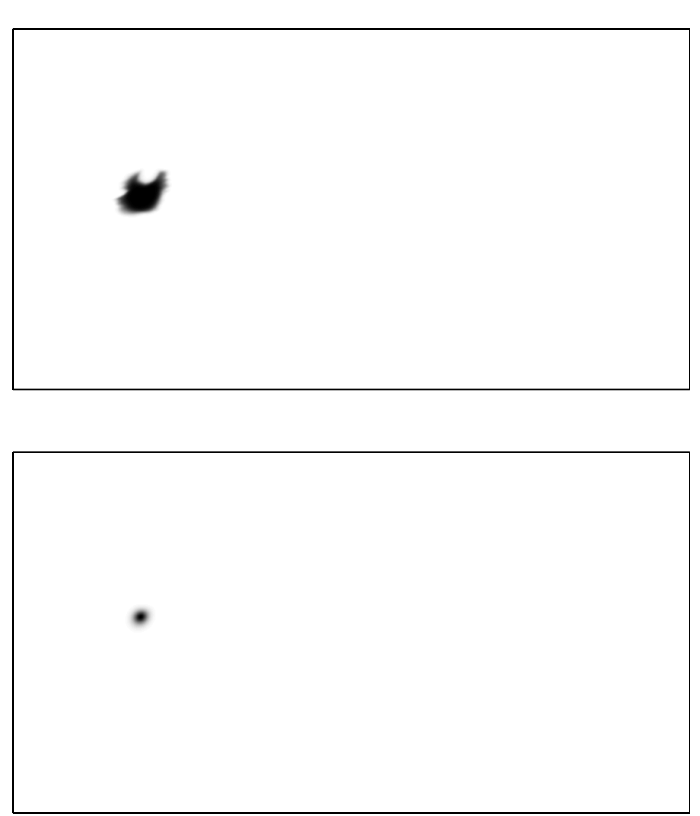

Fig. 6. The association probability $C^{k}(\boldsymbol{x})$ (top) and the posterior position $\mathrm{PMF} X_{\mathrm{s}}^{k}(\boldsymbol{x})$ (bottom) for SEF $k=33$, which is tracking a cyclist, in frame 61 of Neovision2 Tower image sequence 001.

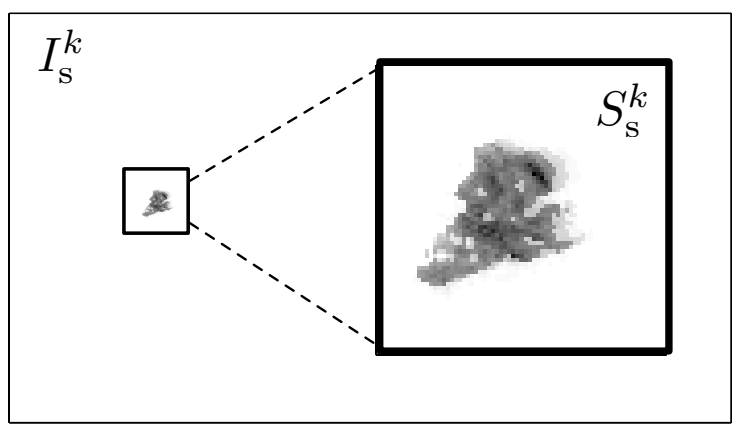

Fig. 7. The posterior image $I_{\mathrm{s}}^{k}(\boldsymbol{i})$, for SEF $k=33$, which is tracking a cyclist, in frame 61 of Neovision2 Tower image sequence 001. The inset shows the corresponding posterior shape $S_{\mathrm{s}}^{k}(\boldsymbol{j})$.

A high degree of match results in a large update of the shape $S_{\mathrm{s}}^{k}(\boldsymbol{j})$, while a low degree of match leads to a small update. The resulting posterior shape is shown for a single SEF in Figure 7

Rather than combining the predicted and measured images, the posterior image $I_{\mathrm{s}}^{k}(\boldsymbol{i})$, is computed according to a maximum a posteriori approach based on the shape and position:

$$
I_{\mathrm{s}}^{k}(\boldsymbol{i})=S_{\mathrm{s}}^{k}(\boldsymbol{j}) \otimes X_{\mathrm{smax}}^{k}(\boldsymbol{x})
$$

The posterior image, which is shown for a single SEF in Figure 7, then provides top-down guidance for new detections according to Eqn. (1) in the object detection stage.

\section{Tracking Output}

Each SEF $k$ outputs the posterior image $I_{\mathrm{s}}^{k}$ of the object that it is tracking. This learned image is then parameterized by calculating its ellipse of second order moments [52]. The $2 \sigma$ length and width along the ellipse major and minor axis, respectively, are used to define an oriented output bounding box for each object, in every frame.

\section{WHAT: OBJECT RECOGNITION}

This section describes the S-CNN and SLFN ensemble classification algorithms, detailing their supervised offline training and application to online object recognition.

While a variety of image classifiers could act as the what processing stream, S-CNNs and SLFNs, in which only the output layer weights are learned, have the advantage of being fast to train (on the order of minutes on standard PCs) and hence are well suited to tasks that require frequent domainspecific re-training.

\section{A. Shallow Convolutional Neural Network}

S-CNN Offline Training: Here we summarise our application-specific S-CNN implementation, while an in depth description of the algorithm may be found in [23]. The network architecture consists of five layers: an input image pixel layer, three hidden unit layers, and an output layer. Only the weights that project to the final layer are learned. The S-CNN can be divided into two conceptual stages: a convolutional filtering and pooling stage formed by the first two hidden layers, which extract translation and scale invariant features, and a classification stage consisting of the third hidden layer and the output layer.

Stage 1: Convolutional Filtering and Pooling. Each domainspecific $\mathrm{S}-\mathrm{CNN}$ is trained on a single batch of image patches of size $61 \times 61$ pixels. The bank of 24 visual processing filters shown in Figure 2, which serve as generic object detectors in the where processing stream, are reused here as the first layer of convolutional filters. Following [23], the first hidden layer units are obtained by applying a termwise nonlinear function $g_{1}(u)=u^{2}$. The first hidden layer activations are averagepooled and down-sampled by applying a uniform low pass filter with a pooling size of $18 \times 18$ pixels and stride of 6 pixels. Finally a termwise nonlinear function of form $g_{2}(u)=u^{0.25}$ is applied to obtain the image features.

Stage 2: Classification. The features from the second hidden layer are concatenated and linearly projected onto 12000 hidden units using a fully-connected set of real-valued input weights, which is set only once during training following the method of [53]. Applying the termwise squaring function $g_{1}(u)$ to every mapped feature yields the third hidden layer activations. Output labels can then be predicted by linearly mapping these activations using a set of fully-connected output weights obtained as in [23], and described further below.

In order to train the $\mathrm{S}-\mathrm{CNN}$, pre-processed (see Section III) $61 \times 61$ pixel image patches are extracted from its training image sequences. Using the Neovision2 Tower training videos, this involves extracting image patches from 15 image sequences $(010-024)$ based on the positions of the ground 
truth bounding boxes. To simulate the effect of object tracking during training, the centre of each patch includes positional Gaussian random jitter about the object ground truth location, with a standard deviation of 10 pixels in both the $x$ and $y$ axis directions. In each patch, the pixels outside a central circular spatial attention region of radius 30 pixels are set to 0 . Additional patches are randomly extracted from background regions in each training image to provide training examples for a background Clutter class. The training examples are then randomly shuffled and the class abundances are balanced so that the number of training examples is uniformly spread among four classes: Car, Person, Cyclist and Clutter.

Given that the convolutional filters, the pooling parameters and the classifier input weights are fixed, the offline training algorithm only involves finding the set of optimal output weights. These are obtained by forming a set of linear equations from a single batch of training class labels and output layer activations and solving for the output weights using least squares regression as in [23].

S-CNN Online Object Recognition: In online processing, raw pixel image patches, which are centered on the position of each SEF, are presented as input to the trained S-CNN in the form of an input vector $\mathbf{x}_{\text {test }}$. Following the matrix notation of [23], the S-CNN output for each patch is the predicted label vector $\mathbf{y}_{\text {test }}$ whose length corresponds to the number of classes:

$$
\mathbf{y}_{\text {test }}=\mathbf{W}_{\text {out }} g_{1}\left(\mathbf{W}_{\text {in }} g_{2}\left(\mathbf{W}_{\text {Pool }} g_{1}\left(\mathbf{W}_{\text {Filter }} \mathbf{x}_{\text {test }}\right)\right)\right),
$$

where the convolution matrices $\mathbf{W}_{\text {Filter }}$ and $\mathbf{W}_{\text {Pool apply }}$ convolutional filtering and pooling, respectively, the matrix $\mathbf{W}_{\text {in }}$ corresponds to the fully-connected input weights, and the matrix $\mathbf{W}_{\text {out }}$ corresponds to the fully-connected output weights. If the S-CNN were used on its own, without applying the SLFN, the predicted class would be given by the index of the maximum value in $\mathbf{y}_{\text {test }}$.

\section{B. Single Hidden Layer Feedforward Network Ensemble}

SLFN Offline Training: We next train SLFNs to predict the ground truth class label associated with each SEF by combining object state (where stream) information and the corresponding S-CNN (what stream) output unit activations. To reduce the potential for over fitting, an ensemble [54] of seven small SLFNs are trained separately. Each SLFN employs the same type of architecture as the S-CNN classification stage. The input features of the first six SLFNs are linearly mapped onto 320 hidden units using a fixed set of fullyconnected input weights that are set randomly only once in training [24]. Using the same approach, the seventh SLFN instead maps the vector form of the $71 \times 71$ pixel posterior shape (e.g. see Figure 7) onto a layer of 12800 hidden units. In all SLFN instances, a termwise logistic sigmoid function $g(u)=1 /(1+\exp (-u))$ is applied to each hidden unit, and these activations are mapped to the output units using an optimal set of fully-connected output weights that is learned during training. As was done for the S-CNN, the optimal output weights for each SLFN are obtained in one shot using least squares regression.
The training procedure for the first six SLFNs relies on a set of 10 features, comprising the softmax of the S-CNN output vector from Eqn. (24) (6 features), and state variables in the form of predicted object bounding box width, length and absolute inclination angle (about the $\mathrm{x}$-axis), as well as the energy of the posterior position PMF: $\sum_{\boldsymbol{x}}\left(X_{\mathrm{s}}^{k}(\boldsymbol{x})\right)^{2}$, which measures the degree to which a SEF has collapsed (or latched) onto its object. Before training the SLFN, each state variable is pre-processed by subtracting the training sample mean and then normalizing by the rms of the entire meansubtracted training sample, and these parameters are saved and also used in online pre-processing. Six SLFNs are then trained using a 65 dimension input feature vector that is formed by multiplying pairs of features, for all unique pairwise combinations plus the individual unpaired features themselves.

In order to accumulate training examples, CACTuS-FL and the S-CNN are applied the Neovision2 Tower training sequences 001, 010, 013, 014, and 017, for which we added unique object IDs by hand to the original ground truth data. This allows optimal associations to be made between SEF bounding boxes and ground truth bounding boxes using the Munkres algorithm [55]. This mapping procedure is used to assign true class labels to each tracked object, which produces the required set of training labels. The first six SLFNs are trained by applying a bagging technique that randomly divides the data among six separate sets. In the case of the posterior shape based (seventh) SLFN, all of the training data is used in a single batch.

SLFN Online Object Recognition: During online processing, given the vector $\mathbf{f}_{\text {test }}^{c}$ of input features appropriate for each trained SLFN $c=1, \ldots, 7$, the output unit vector $\mathbf{y}^{\prime c}$ test is given by:

$$
\mathbf{y}_{\text {test }}^{\prime c}=\mathbf{W}_{\text {out }}^{\prime c} g\left(\mathbf{W}_{\text {in }}^{\prime c} \mathbf{f}_{\text {test }}{ }^{c}\right) \text {, }
$$

where the matrices $\mathbf{W}_{\mathrm{in}}^{\prime c}$ and $\mathbf{W}^{\prime c}$ out correspond to each of SLFN input and trained output weights, respectively. Finally, a softmax function is applied to each output vector, and the SLFNs are used in an ensemble by combining their output through an element-wise sum:

$$
\mathbf{y}_{\text {ensemble }}^{\prime}=\sum_{c=1}^{7} \operatorname{softmax}\left(\mathbf{y}_{\text {test }}^{\prime c}\right)
$$

The class predicted by the online object recognition system is given by the index of the maximum value in $\mathbf{y}^{\prime}$ ensemble.

\section{EXPERIMENTAL EVALUATION}

This section describes the data used in our experiments together with a summary of previous evaluations of the main system components. The section also details our experimental parameters, highlighting any use of prior knowledge, as well as explaining the performance evaluation metrics. The system performance is then compared against existing online object recognition benchmark results [28], while the impact of the main components (CACTuS-FL, S-CNN, SLFN ensemble) on its performance is also investigated. 


\section{A. Previous Experiments}

We summarise previous experimental results for key components of our online object recognition system: generic feature extraction, CACTuS-FL and the S-CNN, using separate visual tracking and image classification benchmarks.

Generic feature extraction: The choice of convolutional filter bank and individual filter size were made based on experiments [40] using the Neovision2 Tower training sequence 001, where the multi-object tracking performance for all object classes was evaluated in terms of the best Recall $(60.37 \%)$ and tracking precision MOTP (43.44\%).

Where-CACTuS-FL: CACTuS-FL was evaluated using 8 videos from the VOT2013 single object tracking benchmark [56]. In these experiments [10] the robustness of the tracker was measured by the number of tracking failures. CACTuS-FL incurred 4 tracking failures, as compared to the well known TLD algorithm [57] that had 39 tracking failures, and the state-of-the-art LGT algorithm [58] that had 2.75 tracking failures. A qualitative evaluation on multi-object tracking using soccer videos was also presented.

What-S-CNN: The S-CNN was previously evaluated [23] on the MNIST [59], NORB [60], SVHN [61] and CIFAR10 [62] benchmark data sets, achieving image classification error rates of $0.37 \%, 2.21 \%, 3.96 \%$ and $24.14 \%$, respectively. In the case of MNIST and NORB, this represents state-ofart image classification accuracy if excluding techniques that perform training set data augmentation [63]. Furthermore, the experiments showed that $\mathrm{S}-\mathrm{CNNs}$ are robust in the sense that the same network metaparameters can be applied across different data sets to yield similar performance to that obtained by tuning the metaparameters for each data set.

\section{B. Online Object Recognition Experiments}

While the key aspects of our online object recognition system have been tested separately, testing the integrated system requires a MOT data set with multiple target classes. As outlined in Section II. existing MOT datasets only exercise tracking of a single class, and often provide precomputed detections [25], [26]. By contrast, we require a multi-object, multi-class benchmark and this is provided by Neovision2 [27]. This set of challenging image sequences, captured under varying environmental conditions, contains numerous targets, including stationary objects, which can undergo occlusions by neighbouring objects or background clutter.

Benchmark Data: The Neovision2 Tower data set consists of 50 training and 50 test videos captured from an elevated camera. In both Tower training and test sets the camera is rotated by $90^{\circ}$ after the first 24 videos, and, given that this changes the ground sample distance (pixel/m), we limit our study to videos $001-024$ in both the training and test sets.

Each image sequence was recorded at 29.97 frames/s and has 871 annotated frames, with ground truth data consisting of a class label and oriented bounding box coordinates for each object of interest. Five target object classes are present in the Tower data domain (Car, Truck, Bus, Person, Cyclist) and, through random sampling of the background, we include a sixth Clutter class in order to identify SEFs that are tracking background objects. Due to the scarcity of Truck and Bus training examples, however, we avoid training and testing on the (few) videos that do contain these object types, which leaves the following four classes: Car, Person, Cyclist, Clutter. Following these criteria and also simply excluding any video found to have clearly incorrect ground truth annotations, we select 12 Neovision 2 Tower test set videos: 001, 002, 009, 010, 012, 013, 017, 018, 019, 021, 022, 023. This set of videos, which contains 82139 ground truth objects across 10452 image frames, was tested only once.

\section{Experimental Parameters}

Prior Knowledge: While the majority of architectural decisions and run time parameter settings for our system were chosen empirically based on previous experiments [6], [10], [23], some were tuned for the Neovision2 Tower training data set. These system parameters constitute domain-specific prior knowledge and are listed in Table I, which outlines the reason behind each choice.

System Initialization: CACTuS-FL is initialized in the first frame of an image sequence by positioning the SEFs at regular intervals in a $14 \times 8$ rectangular grid across the scene. The position, shape and velocity PMFs for each SEF are initialized using isotropic 2D Gaussian distributions.

\section{Performance Evaluation Metrics}

The Neovision 2 object recognition performance metrics [64] are based on the degree of spatial overlap $d_{t, i, k}$ between each ground truth bounding box region $\mathbf{r}_{t, i}^{G T}$ and every candidate bounding box region $\mathbf{r}_{t, k}^{S E F}$ output by the $k^{t h} \mathrm{SEF}$ :

$$
d_{t, i, k}=\frac{\mathbf{r}_{t, i}^{G T} \cap \mathbf{r}_{t, k}^{S E F}}{\mathbf{r}_{t, i}^{G T} \cup \mathbf{r}_{t, k}^{S E F}},
$$

where $t$ refers to the image frame and $i$ is the ground truth index.

To evaluate the online object recognition performance we use the publicly available Neovision2 evaluation tool [64]. This uses the Munkres algorithm [55] to find optimal SEF to ground truth bounding box associations in each frame for a spatial overlap threshold of $T_{d}=0.2$. For each image sequence $s$, the system performance in detecting each target object class $o$ (i.e. Car, Person, Cyclist) is measured using the Normalized Multiple Object Thresholded Detection Accuracy (NMOTDA):

$$
\mathrm{NMOTDA}_{s, o}=1-\frac{\sum_{t}\left(\mathrm{FN}_{t, o}+\mathrm{FP}_{t, o}\right)}{\sum_{t} \mathrm{GT}_{t, o}},
$$

where in each frame $t, \mathrm{GT}_{t, o}, \mathrm{FN}_{t, o}$ and $\mathrm{FN}_{t, o}$ are the number of ground truth objects, false negatives, and false positives, respectively, of object class $o$. NMOTDA is reported as a number in the range $(-\infty, 1]$. The $\mathrm{NMOTDA}_{s, o}$ scores are then aggregated across all image sequences to yield the Weighted Normalized Multiple Object Thresholded Detection Accuracy (WNMOTDA):

$$
\mathrm{WNMOTDA}_{o}=\frac{\sum_{s} \mathrm{NMOTDA}_{s, o} \times \mathrm{GT}_{s, o}}{\sum_{s} \mathrm{GT}_{s, o}},
$$


TABLE I

PRIOR KNOWLEDGE.

\begin{tabular}{|c|c|c|c|}
\hline Parameter & Symbol & Value & Justification \\
\hline Size of learned CRBM convolutional filter & & $16 \times 16$ pixels & Tuned for tracking performance of all objects in the scene [40]. \\
\hline Size of posterior shape & & $71 \times 71$ pixels & $\begin{array}{l}\text { Chosen by eye to ensure that } S_{\mathrm{s}}^{k}(\boldsymbol{j}) \text { is large enough } \\
\text { to encompass and collapse on any person, cyclist or car. }\end{array}$ \\
\hline Total number of SEFs & $\bar{K}$ & 112 & Chosen to encourage competition between SEFs over the entire scene. \\
\hline Size of second order moment ellipse & & $2 \sigma$ & $\begin{array}{l}\text { Chosen so that the bounding boxes of collapsed SEFs } \\
\text { encompass their object, but do not extend too far beyond this. }\end{array}$ \\
\hline Size of image patch & & $61 \times 61$ pixels & $\begin{array}{l}\text { Chosen so that the S-CNN input captures a large fraction of cars, } \\
\text { but limits the extent of the background around people or cyclists. }\end{array}$ \\
\hline
\end{tabular}

where the weight $\mathrm{GT}_{s, o}$ is the total number of ground truth objects belonging to class $o$ that are present in image sequence $s$. Average NMOTDA and Average WNMOTDA are also calculated for all object types according to Eqn. 28) and Eqn. 29, respectively, by ignoring the object class label $o$.

In sequences for which we have added ground truth object IDs, such as 001, we also apply the CLEAR MOT multi-object tracking metrics [65]. Following the implementation of [66], the optimal mapping between SEFs and ground truths is found across all frames in terms of the total spatial overlap. The associated ground truth and SEF pairs are then identified as matches $j \equiv(i, k)$ when $d_{t, j}$ exceeds a user-defined threshold $T_{d}$, which can be varied between 0 and 1 . Figure 8 illustrates some examples of matched $\mathrm{SEF} /$ ground truth pairs for $T_{d}=$ 0.2 . This procedure is used to assign ground truth class labels to SEFs for the purpose of generating SLFN training data.

\section{E. Results}

Table II lists the training and validation classification accuracies obtained by applying the S-CNN to image patches extracted around clutter and randomly jittered ground truth object positions. The un-jittered validation set accuracies obtained here on training video 001 are comparable to the range of accuracies $(96.77 \%-100 \%)$ obtained by a deep CNN [31] on Neovision2 Tower data. The validation results in Table [I] indicate that the classification accuracy of the S-CNN degrades considerably, especially for the Person class, when random position jitter is applied to the image patches, despite the fact that the same approach was used for the training patches.

TABLE II

S-CNN CLASSIFICATION ACCURACY FOR TOWER TRAINING $(010-024)$ AND VALIDATION (001) SEQUENCE IMAGE PATCHES.

Data set Training $(010-024) \quad$ Validation (001) Validation (001)

\begin{tabular}{lccr} 
& with jitter & without jitter & with jitter \\
\hline \hline Car & $99.89 \%$ & $100.00 \%$ & $99.89 \%$ \\
\hline Person & $96.76 \%$ & $95.45 \%$ & $78.42 \%$ \\
\hline Cyclist & $95.52 \%$ & $99.46 \%$ & $96.07 \%$ \\
\hline Clutter & $99.07 \%$ & $97.61 \%$ & $97.67 \%$ \\
\hline
\end{tabular}

In order to gain some intuition into the impact of tracking and classification accuracy on NMOTDA, we attempt to decouple the two effects in Figure 9 , which shows validation results from Tower training sequence 001 . Starting with perfect tracking and classification, NMOTDA is made progressively worse by first classifying using the S-CNN, by next adding position jitter in its input images patches, and finally by also
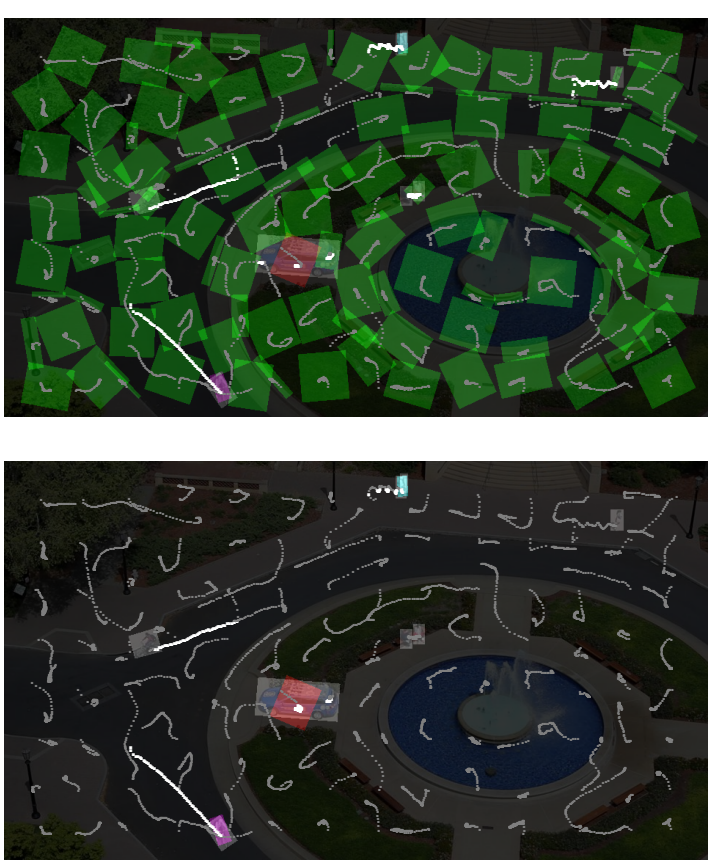

Fig. 8. Neovision2 Tower image sequence 001 frame 61 showing SEF output from the current frame and CLEAR MOT [65] tracks up to and including this frame. CACTuS-FL SEF tracks are shown as grey dots and those identified as SEF and ground truth matches are shown as white dots. Ground truth bounding boxes are indicated by shaded grey rectangles, which are centered on every car, person or cyclist in the scene. Bounding boxes estimated by CACTuS-FL in the current frame, which are computed by parameterizing the object shape learned by each SEF, are shown in green, red, magenta and cyan for SEFs classified as Clutter, Car, Cyclist and Person, respectively. The top plot shows all bounding boxes, while the bottom plot shows only those bounding boxes that have not been classified as Clutter.

adding position jitter to the bounding boxes. Aside from object tracking accuracy, a second key aspect is that none of these four simulated tests incorporate clutter-tracking SEFs, which would provide additional false positives. CACTuS-FL and the S-CNN have the lowest score in Figure 9 for this very reason: operating 112 SEFs across the scene means that the vast majority of SEFs track clutter sources. The S-CNN on its own, with a typical Clutter class accuracy of $\sim 97.6 \%$ (see Table $[\mathrm{II}$ ), would then yield $\sim 2.5$ false positives per frame and thus reduce the NMOTDA score.

This inherent challenge posed by tracking everything motivates the need for a SLFN ensemble. The Tower test results in Figure 10 illustrate this point, where the inclusion of the 

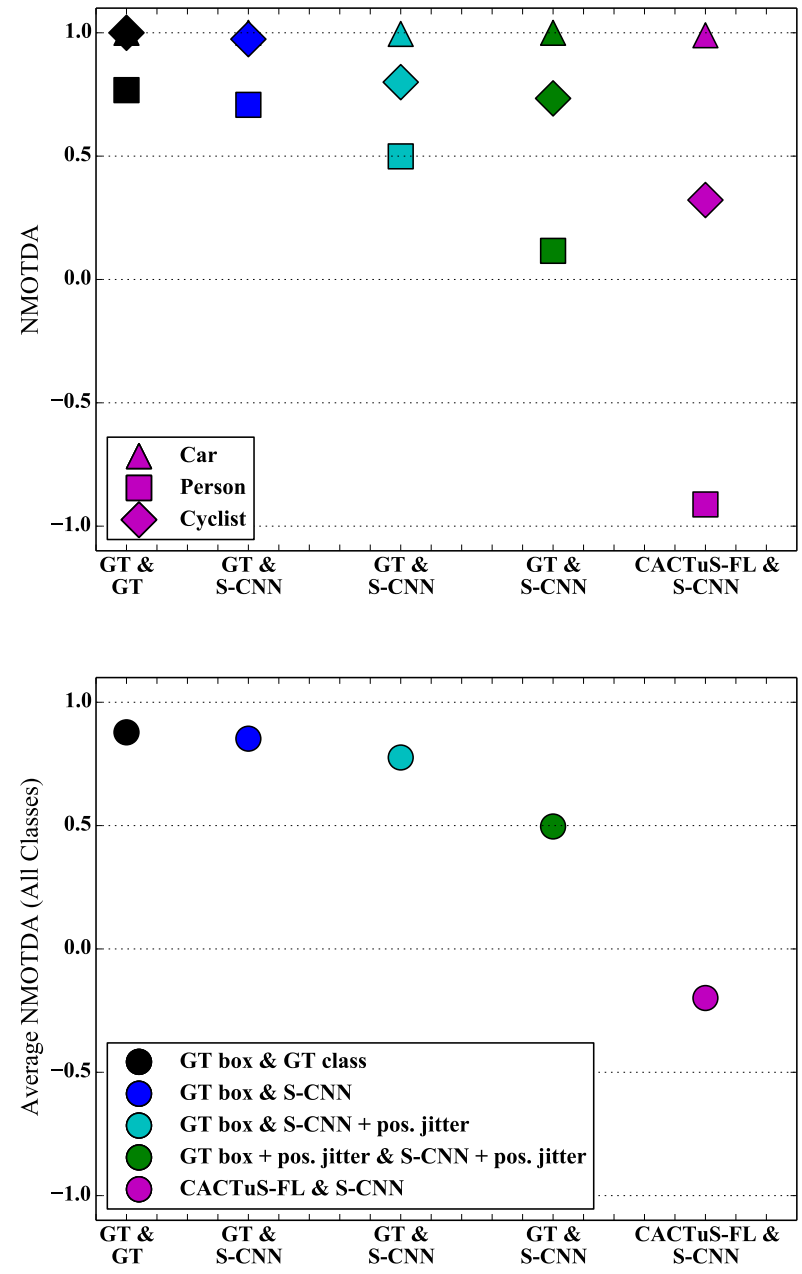

Fig. 9. NMOTDA scores for Neovision2 Tower training sequence 001 used here for validation. The top plot shows NMOTDA for each class, the bottom plot shows the Average NMOTDA scores for all classes. Black markers correspond to taking the ground truth position and class label data as system outputs (e.g. perfect object tracking and classification). Blue markers correspond to perfect tracking and S-CNN based classification. Cyan markers correspond to perfect tracking, but here the S-CNN has random jitter applied to its input image patch positions. Green markers show the case when position jitter is also applied to the ground truth bounding box to simulate the effect of imperfect tracking. In all cases mentioned thus far, the number of SEFs operating in a frame is equal to the number of ground truths in that frame. Magenta markers correspond to tracking using CACTuS-FL and classification using the S-CNN, and in this case 112 SEFs operate in each frame, with the vast majority tracking background clutter objects.

SLFN ensemble greatly improves both the overall and classwise performance. The marked improvement is due to a large reduction in false positives while the number of false negatives tends to remain about the same. Together, the S-CNN and SLFN ensemble fulfil the dual roles of (1) object detection: rejecting Clutter objects while retaining target objects, and (2) object recognition: correctly classifying the target objects (Car, Person, Cyclist), as illustrated by Figure 8.

Table III compares the total numbers of detections, false negatives, and true positives with the total numbers of ground truth objects in our Tower test set of 12 videos. This indicates, for instance, that when considering all objects classes together,
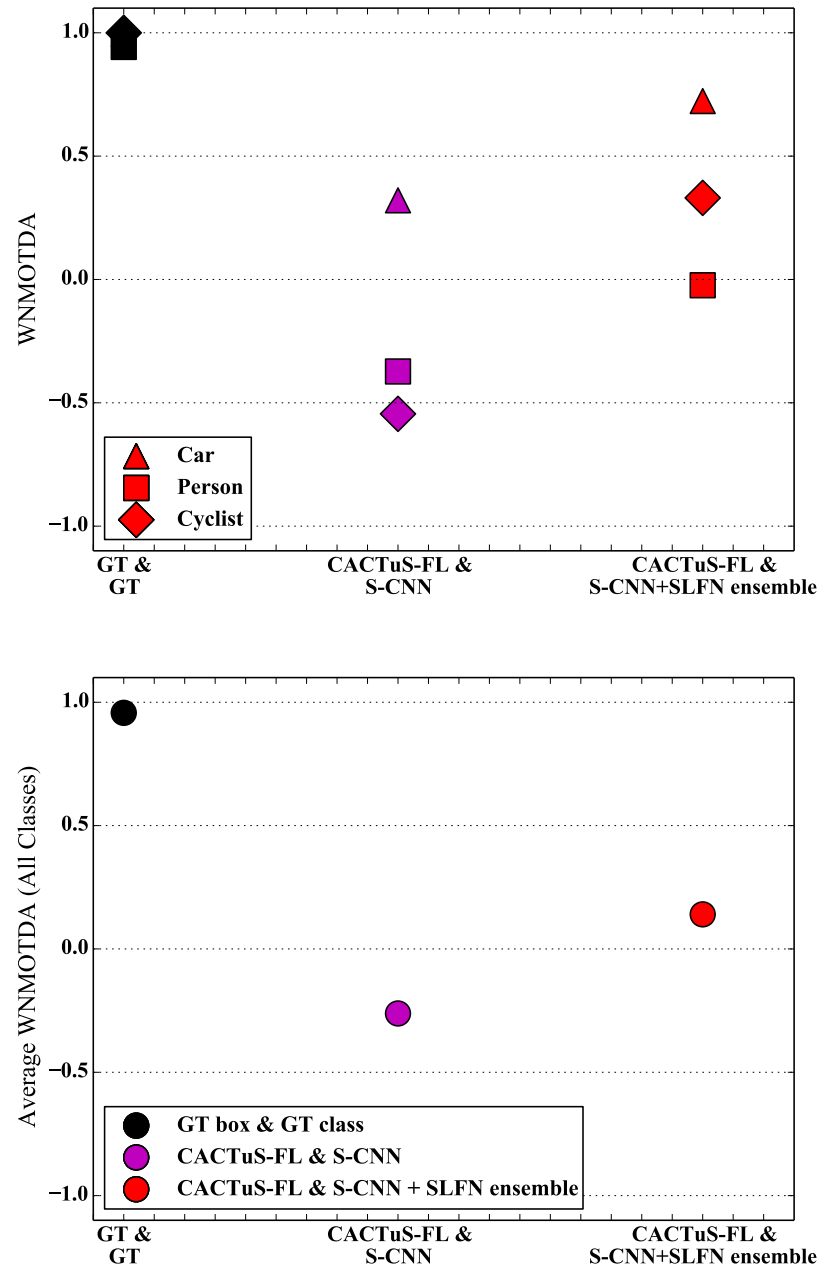

Fig. 10. Neovision2 Tower test WNMOTDA scores, computed across 12 videos. Black markers indicate perfect tracking and classification, magenta markers indicate results from CACTuS-FL \& S-CNN, and red markers indicate results from CACTuS-FL \& S-CNN + SLFN ensemble. The top plot shows the individual class WNMOTDA, while the bottom plot shows the Average WNMOTDA for all classes.

the total Recall is $\sim 41 \%$, while the number of false positives per frame is $\sim 2.09$. In Figure 11 we compare WNMOTDA with data points that we have extracted from the figures in [28]. Here Teams A, B and C rely on Neuromorphic Vision algorithms, whereas those denoted as Baseline are the results of a computer vision algorithm. Our system is competitive with the state-of-the-art [30] (Team A) in terms of the detection score (Average WNMOTDA), which demonstrates the efficacy of our track everything approach. We also achieve the top scores for Cars and Cyclists, although it should be noted that this is on a reduced 12 video test set.

\section{F. Discussion}

Prior knowledge: We have shown that accurate online object recognition can be implemented by using a generalpurpose multi-object tracking system that is able to detect and track all salient objects. For this to work, the use of object 
TABLE III

TOWER TEST SET RESULTS ACROSS ALL 10452 FRAMES: GROUND TRUTHS, DETECTIONS, FALSE NEGATIVES, FALSE POSITIVES.

\begin{tabular}{lcccr} 
& $\sum_{t} \mathrm{GT}_{t}$ & $\sum_{t} \mathrm{Det}_{t}$ & $\sum_{t} \mathrm{FN}_{t}$ & $\sum_{t} \mathrm{FP}_{t}$ \\
\hline \hline All & 82139 & 55271 & 48746 & 21878 \\
\hline Car & 10452 & 11499 & 923 & 1970 \\
\hline Person & 61699 & 35730 & 44558 & 18589 \\
\hline Cyclist & 9988 & 8085 & 4294 & 2391 \\
\hline
\end{tabular}
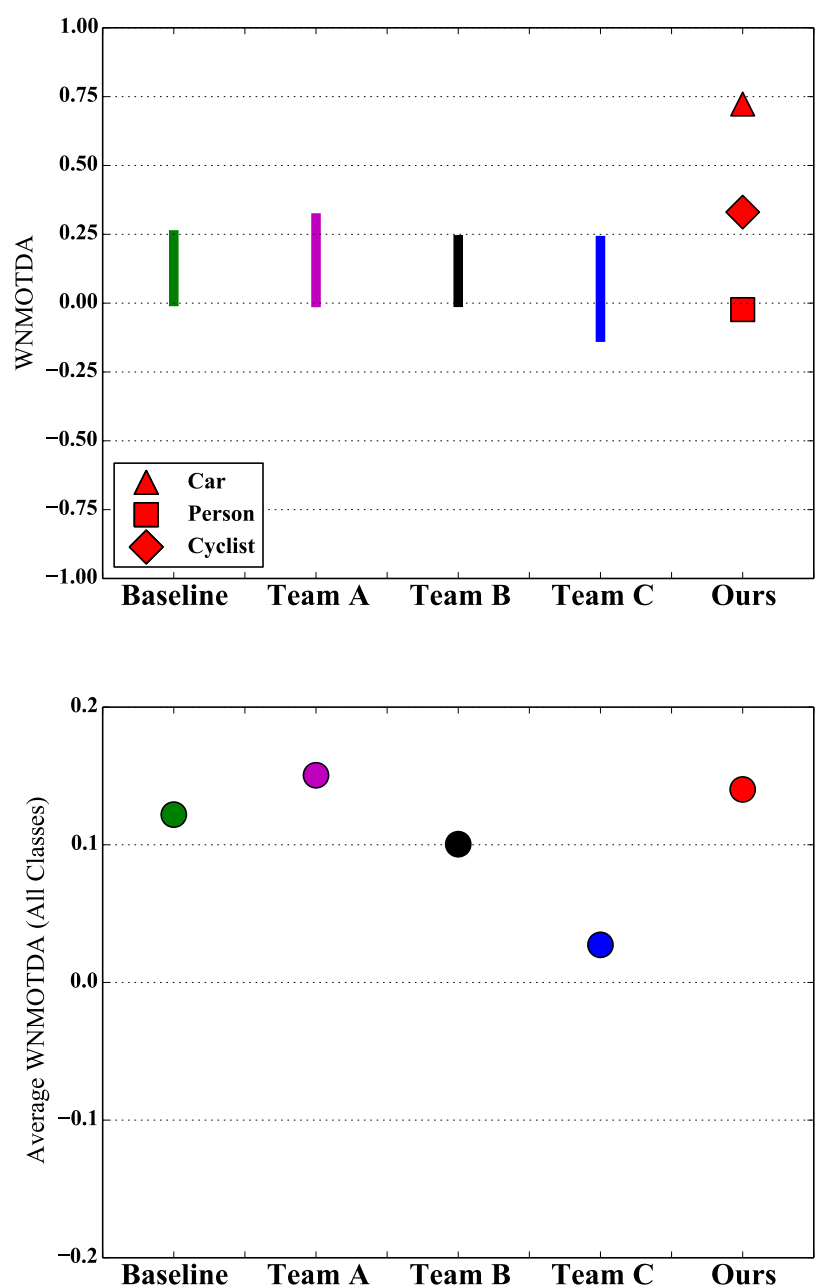

Fig. 11. WNMOTDA published by other teams [28] (Baseline and Teams A-C) and WNMOTDA obtained with our system (CACTuS-FL \& S-CNN + SLFN ensemble) across 12 Neovision2 Tower test sequences (in red). The top plot shows our WNMOTDA scores for individual classes (red symbols), while the vertical coloured bands indicate the approximate range of individual class scores obtained by the competing teams. The bottom plot shows the WNMOTDA detection scores, which are obtained by treating all objects (Car, Person, Cyclist) as a single class. Our approach achieves state-of-the-art performance for Car and Cyclist, and comparable performance for Person. The NMOTDA score for Person is reduced in cases when a single SEF tracks a group of people walking together, see text for details. specific knowledge should be avoided. We have identified in Table I five sources of domain-specific prior knowledge used by CACTuS-FL: the size of convolutional filters, the SEF shape size, the total number of SEFs, the scale of the second order moment ellipse used to define bounding boxes, and the image patch size. However, none of these parameters were tuned for specific object classes, and therefore do not constitute object specific prior knowledge.

Team A [30] achieved state-of-the-art performance using an approach similar to ours, where salient objects are detected and prior knowledge is mostly embedded into the object classifier. The saliency mechanism consists of fusing multiple saliency channels that are created from several individual feature response maps. However, prior knowledge is embedded into some of these saliency channels using the Targeted Contrast Enhancement (TCE) algorithm to create feature response maps that allow them to "easily detect objects with [specified] colors, ... e.g. finding all red cars on the road." Another point of difference is that Team A do not perform tracking, only detection and classification. Instead they embed motion processing as another saliency channel, which detects pixels that appear to be moving in comparison to a (stationary or registered) background scene.

The primary difference between our approach and traditional tracking-by-detection approaches is that prior knowledge of the objects of interest is removed from detection and tracking, and only used for recognition.

Advantages: The advantage is that all objects are tracked and 'explained away', including sources of clutter. This handling of distracting and occluding clutter improves tracking robustness [10]. For instance, when a person (target) walks behind a lamppost (clutter), the SEF tracking the lamppost learns that it is not moving and the competitive attentional mechanisms in CACTuS-FL allow the SEF tracking the person to ignore the observations from the lamppost.

Limitations: One limitation in our current approach is that the tracking system does not know what the extent of a single object is; it simply associates a consistent set of observations (in shape, position and velocity) with a single SEF. For example, people walking together in a group (thus having the same position and velocity) can be efficiently described in the state-space of a single SEF, and thus be considered a single object. This occurs in the Neovision2 Tower test data set video 023 . Here a single SEF tracks a crowd of people and the classifier labels the track a 'Person'. However, the bounding box of the crowd is larger than the ground-truth box of any individual person, thus failing the spatial overlap requirement $d_{t, j}>T_{d}$ from Eqn. (27). This results in both one false positive for the SEF tracking the crowd and many false negatives for the individual people within the crowd, and thus a poor NMOTA score of -0.26 for the video (see supplemental material). This video is a key contributor to the low WNMOTA for the Person class in Figure 11. Furthermore, without this video the overall Average WNMOTA score would be 0.17 rather than its present value of 0.14 .

Integrating what and where: In our architecture low level processing is performed with a common set of convolutional filters (see Figure 2), resulting in a shared set of features for 
the separate what and where processing streams. The what processing stream is performed by the S-CNN, while the where processing stream is performed using CACTuS-FL. By parameterizing elements of the CACTuS-FL state information, it is possible to efficiently re-integrate the what and where processing stream, using the SLFN ensemble. The benefit of the integration is a gain in Average WNMOTDA of 0.4 as shown in Figure 10 This improvement in recognition performance may provide insight into the function of neurons that integrate both the what and where processing streams in the primate visual cortex [67]. Knowing where an object is (tracking) may help recognise what an object is (classification).

\section{CONCLUSION}

We have presented a system for online object recognition that can autonomously locate and recognize multiple types of objects using biologically inspired what and where processing streams. Our overall approach may be characterized as a shift of the use of object-specific prior knowledge out of the where stream and into the what stream. This enables the where stream, which is implemented as a general purpose multiobject tracking algorithm, to locate every salient object in the scene, including sources of occluding or distracting clutter. Online recognition of localized objects is then handled by reintegration of the what and where processing streams. This takes the form of a SLFN ensemble that combines objecttracking state information with class label estimate information from the S-CNN to provide robust object recognition outputs, the performance of which is comparable to the state-of-the-art.

\section{ACKNOWLEDGEMENT}

This work was completed in part with the support provided by the Defence Science and Technology Group, Australian Department of Defence, Commonwealth of Australia.

\section{REFERENCES}

[1] F. Wilson, S. Scalaidhe, and P. Goldman-Rakic, "Dissociation of Object and Spatial Processing Domains in Primate Prefrontal Cortex," Science, vol. 260, no. 5116, pp. 1955-1958, 1993.

[2] A. Torralba and A. A. Efros, "Unbiased look at dataset bias," in Proceedings IEEE Computer Society Conference on Computer Vision and Pattern Recognition (CVPR), 2011, pp. 1521 - 1528.

[3] A. Razavian, H. Azizpour, J. Sullivan, and S. Carlsson, "CNN features off-the-shelf: An astounding baseline for recognition," in IEEE Conference on Computer Vision and Pattern Recognition Workshops (CVPRW), 2014, pp. 512-519.

[4] R. Girshick, J. Donahue, T. Darrell, and J. Malik, "Rich feature hierarchies for accurate object detection and semantic segmentation," in IEEE Conference on Computer Vision and Pattern Recognition (CVPR), 2014, pp. 580-587.

[5] D. Comaniciu, V. Ramesh, and P. Meer, "Kernel-based Object Tracking," IEEE Trans. Pattern Anal. and Mach. Intell., vol. 25, no. 5, pp. 564-577, 2003.

[6] S. Wong, "Advanced correlation tracking of objects in cluttered imagery," in SPIE Acquisition, Tracking, and Pointing XIX, vol. 5810. SPIE, 2005, pp. 158-169.

[7] T. Baker and M. Strens, "Representation of uncertainty in spatial target tracking," in 1998. Proceedings. Fourteenth International Conference on Pattern Recognition, vol. 2. IEEE, 1998, pp. 1339-1342.

[8] S. Wong and D. Kearney, "Relating image, shape, position and velocity in visual tracking," in SPIE Acquisition, Tracking, Pointing, and Laser Systems Technologies XXIII, vol. 7338. SPIE, 2009.
[9] A. Gatt, S. Wong, and D. Kearney, "Combining online feature selection with adaptive shape estimation," in 25th International Conference of Image and Vision Computing New Zealand (IVCNZ), 2010. IEEE, November 2010, pp. 1-8.

[10] S. Wong, A. Gatt, D. Kearney, A. Milton, and V. Stamatescu, "A competitive attentional approach to mitigating model drift in adaptive visual tracking," in The 29th International Conference on Image and Vision Computing New Zealand (IVCNZ'14). ACM, November 2014, pp. 1-6.

[11] J. Yang, P. A. Vela, Z. Shi, and J. Teizer, "Probabilistic multiple people tracking through complex situations," in In Performance Evaluation of Tracking and Surveillance (PETS) workshop at CVPR 2009, 2009, pp. 79-86.

[12] M. D. Breitenstein, F. Reichlin, B. Leibe, E. Koller-Meier, and L. Van Gool, "Online multiperson tracking-by-detection from a single, uncalibrated camera," IEEE Trans. Pattern Anal. Mach. Intell., vol. 33, no. 9, pp. 1820-1833, 2011.

[13] Z. Wu, J. Zhang, and M. Betke, "Online motion agreement tracking," in Proceeding of the 24th British Machine Vision Conference (BMVC), 2013.

[14] S. H. Bae and K. Yoon, "Robust online multi-object tracking based on tracklet confidence and online discriminative appearance learning," in IEEE Conference on Computer Vision and Pattern Recognition (CVPR), 2014, pp. 1218-1225.

[15] J.-P. Jodoin, G.-A. Bilodeau, and N. Saunier, "Urban tracker: Multiple object tracking in urban mixed traffic," in IEEE Winter Conference on pplications of Computer Vision (WACV), 2014, pp. 885-892.

[16] O. Arandjelović, "Contextually learnt detection of unusual motion-based behaviour in crowded public spaces," in Computer and Information Sciences II. Springer, 2011, pp. 403-410.

[17] J. Schmidhuber, "Deep learning in neural networks: An overview," Neural Networks, vol. 61, pp. 85-117, 2015.

[18] G. E. Hinton, S. Osindero, and Y.-W. Teh, "A fast learning algorithm for deep belief nets," Neural Computation, vol. 18, no. 7, pp. 1527-1554, 2006.

[19] Y. LeCun, L. Bottou, Y. Bengio, and P. Haffner, "Gradient-based learning applied to document recognition," Proceedings of the IEEE, vol. 86, no. 11 , pp. 2278-2324, 1998.

[20] A. Krizhevsky, I. Sutskever, and G. E. Hinton, "ImageNet classification with deep convolutional neural networks," in Advances in neural information processing systems, 2012, pp. 1097-1105.

[21] P. Sermanet, D. Eigen, X. Zhang, M. Mathieu, R. Fergus, and Y. LeCun, "OverFeat: Integrated recognition, localization and detection using convolutional networks," in International Conference on Learning Representations (ICLR 2014), 2014.

[22] K. Simonyan and A. Zisserman, "Very deep convolutional networks for large-scale image recognition," CoRR, vol. abs/1409.1556, 2014.

[23] M. D. McDonnell and T. Vladusich, "Enhanced image classification with a fast-learning shallow convolutional neural network," in 2015 International Joint Conference on Neural Networks (IJCNN), July 2015, pp. 1-7.

[24] M. McDonnell, M. D. Tissera, T. Vladusich, A. van Schaik, and J. Tapson, "Fast, simple and accurate handwritten digit classification by training shallow neural network classifiers with the "extreme learning machine' algorithm," PLOS One, vol. 10, pp. e0 134254 (1-20), 2015.

[25] L. Leal-Taixé, A. Milan, I. Reid, S. Roth, and K. Schindler, "Motchallenge 2015: Towards a benchmark for multi-target tracking," arXiv preprint arXiv:1504.01942, 2015.

[26] A. Milan, L. Leal-Taixe, I. Reid, S. Roth, and K. Schindler, "Mot16: A benchmark for multi-object tracking," arXiv preprint arXiv:1603.00831, 2016.

[27] “DARPA Neovision2," http://ilab.usc.edu/neo2/dataset/. 2013, accessed: 2014-06-04.

[28] R. Kasturi, D. Goldgof, R. Ekambaram, R. Sharma, G. Pratt, M. Anderson, M. Peot, M. Aguilar, E. Krotkov, D. Hackett, D. Khosla, Y. Chen, K. Kim, Y. Ran, Q. Zheng, L. Elazary, R. Voorhies, D. Parks, and L. Itti, "Performance evaluation of neuromorphic-vision object recognition algorithms," in 22nd International Conference on Pattern Recognition (ICPR), 2014, pp. 2401-2406.

[29] D. Paiton, S. Brumby, G. Kenyon, G. Kunde, K. Peterson, M. Ham, P. Schultz, and J. George, "Combining multiple visual processing streams for locating and classifying objects in video," in IEEE Southwest Symposium on Image Analysis and Interpretation (SSIAI), 2012, pp. 4952

[30] D. Khosla, Y. Chen, and K. Kim, "A neuromorphic system for video object recognition," Frontiers in Computational Neuroscience, vol. 8, p. 147, 2014. 
[31] Y. Cao, Y. Chen, and D. Khosla, "Spiking deep convolutional neura networks for energy-efficient object recognition," International Journal of Computer Vision, vol. 113, no. 1, pp. 54-66, 2015.

[32] C. Posch, T. Serrano-Gotarredona, B. Linares-Barranco, and T. Delbruck, "Retinomorphic event-based vision sensors: bioinspired cameras with spiking output," Proceedings of the IEEE, vol. 102, no. 10, pp. 1470-1484, 2014

[33] P. A. Merolla, J. V. Arthur, R. Alvarez-Icaza, A. S. Cassidy, J. Sawada, F. Akopyan, B. L. Jackson, N. Imam, C. Guo, Y. Nakamura, B. Brezzo, I. Vo, S. K. Esser, R. Appuswamy, B. Taba, A. Amir, M. D. Flickner, W. P. Risk, R. Manohar, and D. S. Modha, "A million spiking-neuron integrated circuit with a scalable communication network and interface," Science, vol. 345, no. 6197, pp. 668-673, 2014.

[34] M. Danelljan, G. Häger, F. S. Khan, and M. Felsberg, "Convolutional features for correlation filter based visual tracking," in 2015 IEEE International Conference on Computer Vision Workshop ICCV, 2015, pp. 621-629.

[35] C. Ma, J.-B. Huang, X. Yang, and M.-H. Yang, "Hierarchical convolutional features for visual tracking," in Proceedings of the IEEE International Conference on Computer Vision, 2015, pp. 3074-3082.

[36] Z. Yin and R. Collins, "Moving Object Localization in Thermal Imagery by Forward-backward MHI," in Computer Vision and Pattern Recognition Workshop, 2006, 2006, pp. 133-133.

[37] R. Martin and O. Arandjelović, "Multiple-object tracking in cluttered and crowded public spaces," in International Symposium on Visual Computing. Springer, 2010, pp. 89-98.

[38] M. Cimpoi, S. Maji, and A. Vedaldi, "Deep filter banks for texture recognition and segmentation," in IEEE Conference on Computer Vision and Pattern Recognition (CVPR), 2015, pp. 3828-3836.

[39] M. Varma and A. Zisserman, "Texture classification: Are filter banks necessary?" in IEEE Conference on Computer Vision and Pattern Recognition (CVPR), vol. 2. IEEE, 2003, pp. 691-698.

[40] V. Stamatescu, S. Wong, M. McDonnell, and D. Kearney, "Learned filters for object detection in multi-object visual tracking," in Proc. SPIE Automatic Target Recognition XXVI, vol. 9844, 2016.

[41] H. Lee, R. Grosse, R. Ranganath, and A. Ng, "Convolutional deep belief networksfor scalable unsupervised learning of hierarchical representations," in Proceedings of the 26th Annual International Conference on Machine Learning, ser. ICML '09, 2009, pp. 609-616.

[42] B. A. Olshausen and D. J. Field, "Sparse coding with an overcomplete basis set: A strategy employed by v1?" Vision Research, vol. 37, no. 23, pp. 3311-3325, 1997 .

[43] R. Collins, Y. Liu, and M. Leordeanu, "Online selection of discriminative tracking features," IEEE Trans. on Pattern Analysis and Machine Intelligence, vol. 27, no. 10, pp. 1631-1643, October 2005.

[44] V. Mahadevan and N. Vasconcelos, "Biologically inspired object tracking using center-surround saliency mechanisms," IEEE Trans. Pattern Anal. Mach. Intell, vol. 35, no. 3, pp. 541-554, 2013.

[45] N. Vasconcelos, "Feature selection by maximum marginal diversity: optimality and implications for visual recognition." in IEEE Conference on Computer Vision and Pattern Recognition (CVPR), 2003, pp. 762 769.

[46] V. Stamatescu, S. Wong, D. Kearney, I. Lee, and A. Milton, "Mutua information for enhanced feature selection in visual tracking," in Proc. SPIE, Automatic Target Recognition XXV, vol. 9476, 2015, pp. 947603 947 603-11.

[47] A. Bhattacharyya, "On a measure of divergence between two statistical populations defined by their probability distributions," Bulletin of the Calcutta Mathematical Society, vol. 35, pp. 99-109, 1943.

[48] M. Strens and I. Gregory, "Tracking in Cluttered Images," Image and Vision Computing, vol. 21, no. 10, pp. 891-911, 2003.

[49] J. Cho and B. Yun, "Selective-Attention Correlation Measure for Precision Video Tracking," IEICE - Trans. Inf. Syst., vol. E88-D, no. 5, pp. 1041-1049, 2005.

[50] L. Matthews, T. Ishikawa, and S. Baker, "The Template Update Problem," IEEE Trans. Pattern Anal. Mach. Intell, vol. 26, no. 6, pp. 810 $815,2004$.

[51] S. Grossberg, "Adaptive pattern classification and universal recoding: I. Parallel development and coding of neural feature detectors," Biological cybernetics, vol. 23, no. 3, pp. 121-134, 1976.

[52] A. M. Hillas, "Cerenkov light images of EAS produced by primary gamma rays and by nuclei," in Proceedings of the 19th International Cosmic Ray Conference, vol. 3, 1985, pp. 445-448.

[53] W. Zhu, J. Miao, and L. Qing, "Constrained extreme learning machine: A novel highly discriminative random feedforward neural network," in 2014 International Joint Conference on Neural Networks (IJCNN), July 2014, pp. 800-807.
[54] G. Hinton, O. Vinyals, and J. Dean, "Distilling the knowledge in a neural network," arXiv preprint arXiv:1503.02531, 2015.

[55] J. Munkres, "Algorithms for the assignment and transportation problems," Journal of the Society of Industrial and Applied Mathematics, vol. 5, no. 1, pp. 32-38, 1957.

[56] M. K. et al., "The visual object tracking vot2013 challenge results," in The IEEE International Conference on Computer Vision (ICCV) Workshops, June 2013

[57] Z. Kalal, K. Mikolajczyk, and J. Matas, "Tracking-learning-detection," IEEE Trans. Pattern Anal. and Mach. Intell., vol. 34, no. 7, pp. 14091422, 2012.

[58] L. Cehovin, M. Kristan, and A. Leonardis, "Robust visual tracking using an adaptive coupled-layer visual model," IEEE Trans. Pattern Anal. and Mach. Intell., vol. 35, no. 4, pp. 941-953, 2013.

[59] Y. LeCun, C. Cortes, and B. C. J. C., "The MNIST database of handwritten digits," http://yann.lecun.com/exdb/mnist/ 1998, accessed: July 2015.

[60] Y. LeCun, F. J. Huang, and L. Bottou, "Learning methods for generic object recognition with invariance to pose and lighting," in Proceedings IEEE Computer Society Conference on Computer Vision and Pattern Recognition (CVPR), vol. 2, 2004, pp. 97-104.

[61] Y. Netzer, T. Wang, A. Coates, A. Bissacco, B. Wu, and A. Y. Ng, NIPS Workshop on Deep Learning and Unsupervised Feature Learning. See http://ufldl.stanford.edu/housenumbers 2011.

[62] A. Krizhevsky and G. Hinton, "Learning multiple layers of features from tiny images," Master's thesis, Dept of CS, University of Toronto. See http://www.cs.toronto.edu/ kriz/cifar.html 2009.

[63] S. C. Wong, A. Gatt, V. Stamatescu, and M. D. McDonnell, "Understanding data augmentation for classification: when to warp?" in Digital Image Computing: Techniques and Applications (DICTA), 2016 International Conference on. IEEE, 2016, pp. 1-6.

[64] R. Ekambaram, D. Goldgof, and R. Kasturi, "Neovision2 performance evaluation protocol," http://ilab.usc.edu/neo2/dataset/ neovision2-performance-evaluation-protocol.pdf 2012, accessed: 2015-10-23.

[65] K. Bernardin and R. Stiefelhagen, "Evaluating multiple object tracking performance: The clear mot metrics," EURASIP Journal on Image and Video Processing, vol. 2008, pp. 1-10, 2008

[66] A. Andriyenko, K. Schindler, and S. Roth, "Discrete-continuous optimization for multi-target tracking," in IEEE Conference on Computer Vision and Pattern Recognition (CVPR), 2012, pp. 1926-1933.

[67] S. C. Rao, G. Rainer, and E. K. Miller, "Integration of what and where in the primate prefrontal cortex," Science, vol. 276, no. 5313, pp. 821-824, 1997.

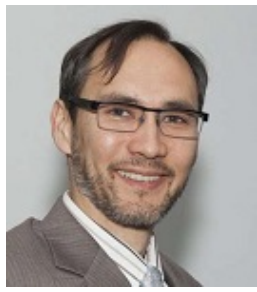

Sebastien Wong (Senior Member, IEEE) joined the Defence Science and Technology (DST) Australia in 1999 where he currently holds the position of Science Team Leader Image Processing. He is also the Director of Machine Learning at Consilium Technology, and an Adjunct Associate Professor at the University of South Australia. Sebastien was awarded the Chief of Air force Gold Commendation in 2008 for his work on missile approach warning algorithms, and a DSTO Achievement Award in 2012 for his work on Hostile Fire Indication algorithms. Sebastien holds a bachelor's degree in Computer Systems Engineering (with honours) from Curtin University, a master's degree in Electronic Systems Engineering and a $\mathrm{PhD}$ in Computer Science, both from the University of South Australia, as well as graduate diploma in Scientific Leadership from the University of Melbourne. Sebastien has published over 20 peer-reviewed papers. His research interests include machine learning algorithms and parallel processing architectures for autonomous vision systems. He is IEEE senior member and a past chair for the IEEE South Australia Section Computer Society Chapter. 


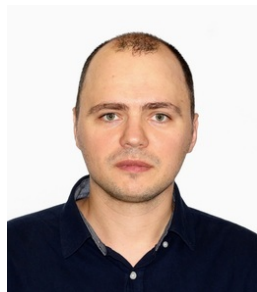

Victor Stamatescu (Member, IEEE) received a Bachelor of Science (Hons), majoring in Physics, and a PhD in Astrophysics, both from the University of Adelaide in 2004 and 2010, respectively. Between 2010 and 2013 he was a postdoctoral research scientist at the Institute of High Energy Physics (IFAE) in Barcelona, Spain. From 2013 to 2014 he was a research fellow in the High Energy Astrophysics group at the University of Adelaide. Since 2014 he has been a research fellow in the School of Information Technology and Mathematical Sciences at the University of South Australia. Victor has published 79 peer-reviewed journal and conference papers. His current research interests are in the areas of visual tracking, image classification and machine learning.

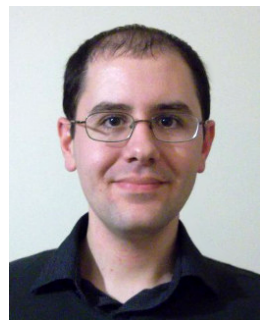

Adam Gatt (Member, IEEE) is a software developer with the Australian Department of Defence. His research interests involve visual tracking, feature learning and tracker evaluation. Adam was awarded a $\mathrm{PhD}$ in Computer Science from the University of South Australia in 2013. He received a bachelor's degree in Information Technology with first class honours in 2008, and was awarded the Joyner Scholarship and UniSA Vice Chancellor and President's Scholarship in 2009. He is a member of the IEEE Computer Society and is a past chair for the South

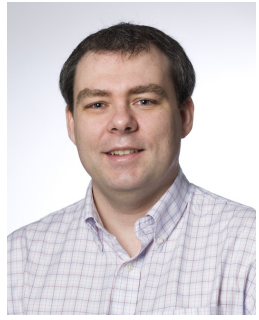

Mark D. McDonnell (Senior Member, IEEE) received the B.E. degree in electronic engineering, the B.Sc. degree (with first class honors) in applied mathematics, and the Ph.D. degree in electronic engineering from The University of Adelaide, Australia, in 1998, 2001, and 2006, respectively. $\mathrm{He}$ is currently Associate Professor, and Principal Investigator of the Computational Learning Systems Laboratory at the University of South Australia, which he joined in 2007. He has published over 100 papers, including several review articles, and a book on stochastic resonance, published by Cambridge University Press. Prof. McDonnell has served as a Guest Editor for Proceedings of the IEEE and Frontiers in Computational Neuroscience. His research interests lie in the intersection between machine learning and neurobiological learning, with a specific focus on the influence of random noise on learning. His contributions to this area have been recognized by the award of an Australian Research Fellowship from the Australian Research Council in 2010, and a South Australian Tall Poppy Award for Science in 2008. He has served as Vice President and Secretary of the IEEE South Australia Section Joint Communications and Signal Processing Chapter.

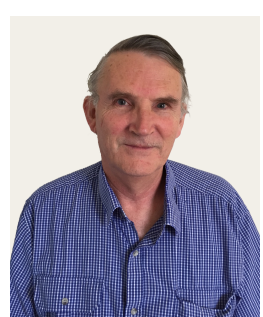

David Kearney is Associate Professor of Computer Science at the University of South Australia. David's research has focused on high performance parallel computing using reconfigurable hardware based on field programmable gate arrays, leading to over 90 refereed publications. His research outputs include the Hardware Join Java language and the ReconfigME operating system for reconfigurable computing. He has interests in applications related to high speed parallel image processing, simulation and tracking. David has in recent years taken an interest in forms of parallel computing inspired by biology including membrane computing and neural networks.

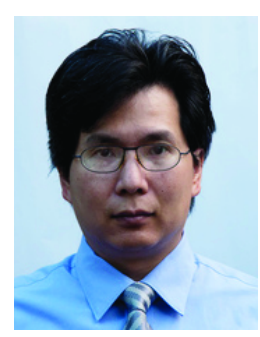

Ivan Lee (Senior Member, IEEE) received BEng, MCom, MER, and $\mathrm{PhD}$ degrees from the University of Sydney, Australia. He was a software development engineer at Cisco Systems, a software engineer at Remotek Corporation, and an Assistant Professor at Ryerson University. Since 2008, he has been a Senior Lecturer at the University of South Australia. His research interests include multimedia systems, medical imaging, data analytics, and computational economics. 